\title{
Holographic trace anomaly and local renormalization group
}

\author{
Srivatsan Rajagopal, ${ }^{a}$ Andreas Stergiou ${ }^{b}$ and Yechao $\mathbf{Z h u}^{c}$ \\ ${ }^{a}$ Center for Theoretical Physics, Massachusetts Institute of Technology, \\ Cambridge, Massachusetts 02139, U.S.A. \\ ${ }^{b}$ Department of Physics, Yale University, \\ New Haven, CT 06520, U.S.A. \\ ${ }^{c}$ Department of Physics, Massachusetts Institute of Technology, \\ Cambridge, Massachusetts 02139, U.S.A. \\ E-mail: srivat91@mit.edu, andreas.stergiou@yale.edu, eltonzhu@mit.edu
}

ABSTRACT: The Hamilton-Jacobi method in holography has produced important results both at a renormalization group (RG) fixed point and away from it. In this paper we use the Hamilton-Jacobi method to compute the holographic trace anomaly for four- and sixdimensional boundary conformal field theories (CFTs), assuming higher-derivative gravity and interactions of scalar fields in the bulk. The scalar field contributions to the anomaly appear in CFTs with exactly marginal operators. Moving away from the fixed point, we show that the Hamilton-Jacobi formalism provides a deep connection between the holographic and the local RG. We derive the local RG equation holographically, and verify explicitly that it satisfies Weyl consistency conditions stemming from the commutativity of Weyl scalings. We also consider massive scalar fields in the bulk corresponding to boundary relevant operators, and comment on their effects to the local RG equation.

KEYwORDS: Renormalization Group, AdS-CFT Correspondence

ARXIV EPRINT: 1508.01210 


\section{Contents}

1 Introduction 1

2 Higher-derivative dilaton gravity 3

3 Trace anomaly from the flow equation $\quad 11$

$\begin{array}{lll}3.1 & \text { Four-dimensional trace anomaly } & 12\end{array}$

$\begin{array}{lll}3.2 & \text { Six-dimensional trace anomaly } & 12\end{array}$

$\begin{array}{lll}3.3 \text { Bounds } & 14\end{array}$

4 Trace anomaly away from fixed points $\quad \mathbf{1 5}$

4.1 Marginal operators 16

$\begin{array}{lll}4.2 & \text { Relevant operators } & 18\end{array}$

$\begin{array}{ll}\text { A ADM formalism } & 20\end{array}$

$\begin{array}{ll}\text { B Boundary terms } & 22\end{array}$

C Expressions for six-dimensional curvature tensors $\quad 24$

D Anomaly coefficients and consistency conditions in $d=4 \quad 24$

\section{Introduction}

In a conformal field theory (CFT) defined in flat space the trace of the stress-energy tensor vanishes. Despite this, in an even-dimensional CFT considered in curved space there is an anomaly and the stress-energy tensor is no longer traceless due to curvature contributions [1]. This trace anomaly has been extensively studied in field theory and beyond (see [2] for a nice review), and, soon after the discovery of the AdS/CFT correspondence [3-5], it was also considered in the context of holography. In particular, Henningson and Skenderis (HS) provided a holographic derivation of the trace anomaly of the boundary two- four- and six-dimensional CFT [6] by studying the divergences of the supergravity action close to the AdS boundary. The four-dimensional result was also obtained later by de Boer, Verlinde and Verlinde (dBVV) using the Hamiltonian formulation of gravity and Hamilton-Jacobi theory $[7,8]$, while the $d=6$ result was obtained using the dBVV method in [9]. Additionally, the dBVV method was used in [10] to compute the $d=8$ holographic anomaly. The Hamilton-Jacobi method was also considered by Papadimitriou and Skenderis [11, 12].

The results of HS and dBVV were obtained with Einstein gravity in the bulk, but this was extended to higher-derivative gravity in $[13,14]$ and [15]. A scalar field $\phi$ has also been considered in the bulk with its kinetic term, while more general situations involving also an axion have been examined in $[16,17]$. The flat space limit of the corresponding contributions to the anomaly has been considered in [18] in any even dimension, while the 
full curvature-dependent contributions have been computed in four dimensions in [9]. The $\phi$-dependent terms for massless $\phi$ correspond to contributions in CFTs with conformal manifolds, and in $d=4$ they take the form of the Paneitz operator in $d=4$ [19], an operator first discussed by Fradkin and Tseytlin [20-22] and also considered by Riegert [23]. The form of these contributions is dictated by conformal covariance [24]. For general even dimension the conformal covariance properties of the $\phi$-dependent part of the holographic trace anomaly were pointed out in [11, 12]. The case of massive $\phi$ has been considered in [25].

In this work we extend these results in $d=4,6$ by considering higher-derivative gravity in the bulk, with higher-derivative quadratic interactions of a scalar field. Our bulk action is given in (2.1) below, and we also consider the required boundary action (2.3). Following the method of [15] we obtain our results for the trace anomaly in equations (3.9) and (3.14) in $d=4,6$ respectively. In $d=4$ the new higher-derivative interactions of the scalar contribute to the trace anomaly in accord with the Paneitz operator [19-23], just like the standard kinetic term in the bulk. In $d=6$ the higher-derivative gravity terms and the standard kinetic term of $\phi$ have not been considered before. For the $\phi$-dependent contributions we find that the kinetic term gives rise to the Branson operator [26], a conformally covariant operator defined in $d=6$, while the higher-derivative terms contribute to the Branson operator, but also give rise to two more conformally covariant operators quadratic in $\phi$ and involving the Weyl tensor. These operators were shown to appear in CFTs with marginal operators in $d=6$ in [27].

The trace anomaly in $d=6$ CFTs in curved space contains three Weyl invariant contributions, with coefficients $c_{1}, c_{2}, c_{3}$, as well as the Euler term, with coefficient $a$. The parameter $c_{3}$ appears in the two-point function of the stress-energy tensor two-point function in flat space, while $c_{1}, c_{2}$ show up in the three-point function. Using positivity of energy flux in lightlike directions it was shown in [28] that one can obtain bounds on the parameters appearing in the three-point function of the stress-energy tensor. These bounds were understood holographically in [29], where they were shown to arise by causality considerations in the bulk. For the $d=6$ case these bounds were considered in [30-32] for Gauss-Bonnet gravity in the bulk. In this paper we extend the result of [30-32] for general higher-derivative gravity in the bulk using our result (3.14).

Outside a conformal fixed point, Osborn has introduced a systematic treatment of the trace anomaly incorporating efficiently renormalization effects of composite operators [33]. In Osborn's analysis a background metric $\gamma_{\mu \nu}$ is introduced and the couplings $g^{I}$ are promoted to spacetime-dependent sources for the corresponding composite operators $\mathcal{O}_{I}$. Besides curvature-dependent counterterms required for finiteness, one needs to also consider counterterms containing derivatives on $g^{I}[34,35]$. Then, a local renormalization group (RG) equation can be derived, valid along the RG flow. This corresponds to a local version of the Callan-Symanzik equation, and yields an expression of the form

$$
T^{\mu}{ }_{\mu}=\beta^{I} \mathcal{O}_{I}+\left(\text { terms with derivatives on } \gamma_{\mu \nu}, g^{I}\right)
$$

for the trace of the stress-energy tensor $T_{\mu \nu}$. The terms with derivatives on $\gamma_{\mu \nu}$ and $g^{I}$ in (1.1) contain coefficients which may be related to flat-space correlation functions involving $T_{\mu \nu}$ and $\mathcal{O}_{I}$. 
Osborn further considered (1.1) and the response of the field theory under Weyl scalings, and derived consistency conditions in $d=2,4$ stemming from the Abelian nature of the Weyl group. These are similar to the well-known Wess-Zumino consistency conditions [36], and have been a subject of interest recently in $d=4[24,37]$, as well as in $d=6$ and more generally in any even $d$ [38]. The main driving force has been a consistency condition of the form

$$
\mu \frac{d \tilde{a}}{d \mu}=G_{I J} \beta^{I} \beta^{J}
$$

where $\mu$ is the RG scale, found by Osborn in $d=2,4$ and shown in [38] to appear in any even $d$. Equation (1.2) ties the monotonicity of the RG flow of a quantity $\tilde{a}$, related to the coefficient of the Euler term in the trace anomaly, to the sign of a symmetric tensor $G_{I J}$. In $d=2$ a positive-definite $G_{I J}$ was found by Osborn [33], thus rederiving Zamolodchikov's $c$-theorem [39], while in $d=4$ only a perturbative analog was obtained [34]. In $d=6$ the sign of $G_{I J}$ was found to be negative in multiflavor $\phi^{3}$ theory $[35,40]$.

Despite their obvious interest from the field theoretic point of view, Osborn's local RG and consistency conditions have received limited attention from the holographic side. Erdmenger developed the subject to some extent in [41], but the results derived there do not illustrate the deep connection of Osborn's formalism with the dBVV formulation of the holographic RG. In this paper we show that the flow equation of dBVV contains Osborn's local RG equation. In $d=4$ and with Einstein gravity and a massless scalar field in the bulk we compute holographically quantities in the local RG equation (1.1) like $\tilde{a}$ and $G_{I J}$ mentioned above. These quantities are related to the local divergent part of the supergravity action close to the boundary. Furthermore, we verify that all Weyl consistency conditions derived by Osborn in $d=4$ are satisfied by the holographic result. We also consider bulk massive scalar fields, and comment on their contributions the anomaly.

This paper is organized as follows. In the next section we describe for completeness the formalism of dBVV in the higher-derivative case. We derive all necessary results needed for the computation of the holographic trace anomaly in section 3. In section 4 we illustrate the relation of the holographic RG to the local RG, and derive an expression for the holographic trace anomaly away from the fixed point. In this section we also comment on the $a$-theorem-like consistency condition (1.2) in $d=4,6$, and discuss the effects on the anomaly originating from massive scalar fields in the bulk. We also include appendices on details of the ADM decomposition, the boundary terms, definitions of curvature tensors in $d=6$, and results in $d=4$ for the coefficients of the anomaly terms away from the fixed point assuming Einstein gravity and a kinetic term for the massless scalar field $\phi$ in the bulk.

\section{Higher-derivative dilaton gravity}

We consider classical dilatonic gravity on an asymptotically-AdS manifold $M_{d+1}$ with metric $\tilde{g}_{\mu \nu}$. The bulk action is taken to be

$$
S_{B}=\int_{M_{d+1}} d^{d+1} x \sqrt{\tilde{g}}\left(\mathscr{L}_{B}^{\tilde{g}}+\mathscr{L}_{B}^{\phi}\right)
$$


where $\tilde{g}$ is the determinant of $\tilde{g}_{\mu \nu}$ and

$$
\begin{aligned}
& \mathscr{L}_{B}^{\tilde{g}}=2 \Lambda-R-a R^{2}-b R^{\mu \nu} R_{\mu \nu}-c R^{\mu \nu \rho \sigma} R_{\mu \nu \rho \sigma}, \\
& \mathscr{L}_{B}^{\phi}=\frac{1}{2} \partial^{\mu} \phi \partial_{\mu} \phi+e R \partial^{\mu} \phi \partial_{\mu} \phi+f R^{\mu \nu} \partial_{\mu} \phi \partial_{\nu} \phi+g \nabla^{2} \phi \nabla^{2} \phi+h \nabla^{\mu} \partial^{\nu} \phi \nabla_{\mu} \partial_{\nu} \phi .
\end{aligned}
$$

Here we allow terms quadratic in $\phi$ with up to two derivatives on $\phi$. The manifold $M$ has a $d$-dimensional boundary $\partial M$ and we also have the boundary action

$$
S_{\partial}=\int_{(\partial M)_{d}} d^{d} y \sqrt{\tilde{h}}\left(\mathscr{L}_{\partial}^{\tilde{h}}+\mathscr{L}_{\partial}^{\phi}\right)
$$

where $\tilde{h}_{i j}$ is the induced metric and

$$
\begin{aligned}
& \mathscr{L}_{\partial}^{\tilde{h}}=2 K+x_{1} R K+x_{2} R^{i j} K_{i j}+x_{3} K^{3}+x_{4} K K^{i j} K_{i j}+x_{5} K_{j}^{i} K^{j}{ }_{k} K^{k}{ }_{i}, \\
& \mathscr{L}_{\partial}^{\phi}=y_{1} K \partial^{i} \phi \partial_{i} \phi+y_{2} K^{i j} \partial_{i} \phi \partial_{j} \phi+y_{3} £_{n} \phi \nabla^{2} \phi,
\end{aligned}
$$

where $K_{i j}$ is the extrinsic curvature, $K=\tilde{h}^{i j} K_{i j}$, and $£_{n}$ is the Lie derivative along the vector $n^{\mu}$ normal to the boundary. The first term in $\mathscr{L}_{\partial}^{\phi}$ is the Gibbons-Hawking-York term for Einstein gravity $[42,43]$. More comments on the boundary terms can be found in appendix B. The case with $e=f=g=h=y_{1}=y_{2}=y_{3}=0$ has been considered in $[15,44]$.

It is straightforward to work out the ADM form [45] of the action

$$
S=S_{B}-S_{\partial} .
$$

Using technology summarized in appendix A we can determine

$$
S=\int d r \int d^{d} y \sqrt{\tilde{h}} \mathscr{L}, \quad \mathscr{L}=\mathscr{L}_{0}^{\tilde{h}}+\mathscr{L}_{0}^{\phi}+\mathscr{L}_{1}^{\tilde{h}}+\mathscr{L}_{1}^{\phi},
$$

where the radial coordinate $r$ is identified with the RG parameter of the boundary theory and

$$
\begin{aligned}
\frac{1}{N} \mathscr{L}_{0}^{\tilde{h}}= & 2 \Lambda-R-K^{2}+K^{i j} K_{i j}, \quad \frac{1}{N} \mathscr{L}_{0}^{\phi}=\frac{1}{2}\left(\partial^{i} \phi \partial_{i} \phi+\left(£_{n} \phi\right)^{2}\right) \\
\frac{1}{N} \mathscr{L}_{1}^{\tilde{h}}= & -a R^{2}-b R^{i j} R_{i j}-c R^{i j k l} R_{i j k l} \\
& +\left(\left(2 a-x_{1}\right) K^{2}-2\left(3 a-x_{1}\right) K^{i j} K_{i j}\right) R \\
& +\left(\left(2 b+2 x_{1}-x_{2}\right) K K^{i j}-2\left(2 b+4 c-x_{2}\right) K_{k}^{i} K^{k j}\right) R_{i j}+2\left(6 c+x_{2}\right) K^{i k} K^{j l} R_{i j k l} \\
& -\left(a+x_{3}\right) K^{4}+\left(6 a-b+6 x_{3}-x_{4}\right) K^{2} K^{i j} K_{i j}-\left(9 a+b+2 c-2 x_{4}\right)\left(K^{i j} K_{i j}\right)^{2} \\
& +\left(4 b+4 x_{4}-x_{5}\right) K K_{j}^{i} K^{j}{ }_{k} K^{k}{ }_{i}-2\left(2 b+c-3 x_{5}\right) K_{j}^{i} K^{j}{ }_{k} K^{k}{ }_{l} K_{i}^{l} \\
& +2\left(b+x_{1}\right) K \nabla^{2} K+\left(8 c+x_{2}\right) K^{i j} \nabla^{2} K_{i j} \\
& -\left(4 b+2 x_{1}-x_{2}\right) K^{i j} \nabla_{i} \partial_{j} K+2\left(b-4 c-x_{2}\right) K^{i j} \nabla_{j} \nabla_{k} K^{k}{ }_{i} \\
& -\left((4 a+b) \tilde{h}^{i j} \tilde{h}^{k l}+(b+4 c) \tilde{h}^{i k} \tilde{h}^{j l}\right) L_{i j} L_{k l} \\
& +\left(\left(4 a-x_{1}\right) R \tilde{h}^{i j}+\left(2 b-x_{2}\right) R^{i j}\right) L_{i j} \\
& -\left(\left(4 a+3 x_{3}\right) K^{2}-\left(12 a+2 b-x_{4}\right) K^{i j} K_{i j}\right) L \\
& -\left(2\left(b+x_{4}\right) K K^{i j}-\left(4 b+8 c-3 x_{5}\right) K_{k}^{i} K^{k j}\right) L_{i j},
\end{aligned}
$$


and

$$
\begin{aligned}
\frac{1}{N} \mathscr{L}_{1}^{\phi}= & e R \partial^{i} \phi \partial_{i} \phi+f R^{i j} \partial_{i} \phi \partial_{j} \phi+g \nabla^{2} \phi \nabla^{2} \phi+h \nabla^{i} \partial^{j} \phi \nabla_{i} \partial_{j} \phi \\
& -\left(\left(e+y_{1}\right) K^{2}-\left(3 e+2 y_{1}\right) K^{i j} K_{i j}+\left(2 e+y_{1}\right) L\right) \partial^{k} \phi \partial_{k} \phi \\
& -\left(\left(f-2 y_{1}+y_{2}\right) K K^{i j}-2\left(f+h+2 y_{2}\right) K_{k}^{i} K^{k j}+\left(f+y_{2}\right) L^{i j}\right) \partial_{i} \phi \partial_{j} \phi \\
& +\left(e R-(e-g) K^{2}+(3 e+f+h) K^{i j} K_{i j}-(2 e+f) L\right)\left(£_{n} \phi\right)^{2} \\
& +2 g K £_{n} \phi\left(£_{n} £_{n} \phi-£_{a} \phi\right)+(g+h)\left(£_{n} £_{n} \phi-£_{a} \phi\right)^{2} \\
& +2(f+g) K \nabla^{2} \phi £_{n} \phi-2(f-h) K^{i j} \nabla_{i} \partial_{j} \phi £_{n} \phi \\
& +\left(2 g+y_{3}\right) \nabla^{2} \phi\left(£_{n} £_{n} \phi-£_{a} \phi\right)+\left(2 h-y_{3}\right) \partial^{i} £_{n} \phi \partial_{i} £_{n} \phi \\
& +\left(2 f-2 y_{1}-y_{3}\right) K \partial^{i} \phi \partial_{i} £_{n} \phi-2\left(f+2 h+y_{2}-y_{3}\right) K^{i j} \partial_{i} \phi \partial_{j} £_{n} \phi .
\end{aligned}
$$

In (2.8) and (2.9) we neglect terms in the right-hand side that are total derivatives.

For the higher-derivative Lagrangian $\mathscr{L}$ of (2.6) we consider the canonical variables $g_{i j}, K_{i j}, \phi, \Sigma=£_{n} \phi, \pi_{i j}, P_{i j}, \pi_{\phi}$, and $P_{\Sigma}$, with the usual definitions

$$
\pi_{i j}=\frac{\partial \mathscr{L}}{\partial \dot{\tilde{h}}^{i j}}, \quad P_{i j}=\frac{\partial \mathscr{L}}{\partial \dot{K}^{i j}}, \quad \pi_{\phi}=\frac{\partial \mathscr{L}}{\partial \dot{\phi}}, \quad P_{\Sigma}=\frac{\partial \mathscr{L}}{\partial \dot{\Sigma}} .
$$

Since $L_{i j}$ is linear in $\dot{K}_{i j}$ it is easy to compute

$$
\begin{aligned}
P^{i j}= & -2\left((4 a+b) \tilde{h}^{i j} \tilde{h}^{k l}+(b+4 c) \tilde{h}^{i k} \tilde{h}^{j l}\right) L_{k l} \\
& +\left(\left(4 a-x_{1}\right) R-\left(4 a+3 x_{3}\right) K^{2}+\left(12 a+2 b-x_{4}\right) K^{k l} K_{k l}\right) \tilde{h}^{i j} \\
& +\left(2 b-x_{2}\right) R^{i j}-2\left(b+x_{4}\right) K K^{i j}+\left(4 b+8 c-3 x_{5}\right) K_{k}^{i} K^{k j} \\
& -\left((2 e+f) \Sigma^{2}+\left(2 e+y_{1}\right) \partial^{k} \phi \partial_{k} \phi\right) \tilde{h}^{i j}-\left(f+y_{2}\right) \partial^{i} \phi \partial^{j} \phi .
\end{aligned}
$$

Equation (2.11) is solved for $L_{i j}$ by

$$
L_{i j}=L_{i j}^{\prime}\left(P-P^{\phi}\right), \quad P_{i j}^{\phi}=-\left((2 e+f) \Sigma^{2}+\left(2 e+y_{1}\right) \partial^{k} \phi \partial_{k} \phi\right) \tilde{h}_{i j}-\left(f+y_{2}\right) \partial_{i} \phi \partial_{j} \phi,
$$

where

$$
\begin{aligned}
L_{i j}^{\prime}(P)= & -\frac{1}{2(b+4 c)}\left(P_{i j}-\left(2 b-x_{2}\right) R_{i j}+2\left(b+x_{4}\right) K K_{i j}-\left(4 b+8 c-3 x_{5}\right) K_{i k} K_{j}^{k}\right) \\
& +\frac{1}{2(b+4 c)(d(4 a+b)+b+4 c)}((4 a+b) P \\
& -\left(2 b^{2}+4 a(b-4 c)+(b+4 c) x_{1}-(4 a+b) x_{2}\right) R \\
& +\left(2 b^{2}+4 a(b-4 c)-3(b+4 c) x_{3}+2(4 a+b) x_{4}\right) K^{2} \\
& \left.-\left(2 b^{2}+4 a(b-4 c)+(b+4 c) x_{4}-3(4 a+b) x_{5}\right) K^{k l} K_{k l}\right) \tilde{h}_{i j} .
\end{aligned}
$$

Also, since $£_{n} \Sigma$ is linear in $\dot{\Sigma}$ we find

$$
P_{\Sigma}=2(g+h)\left(£_{n} \Sigma-£_{a} \phi\right)+2 g\left(\nabla^{2} \phi+K \Sigma\right),
$$

which allows us to express

$$
\dot{\Sigma}=N\left(\frac{1}{2(g+h)}\left(P_{\Sigma}-2 g\left(\nabla^{2} \phi+K \Sigma\right)\right)+£_{a} \phi\right)+£_{N} \Sigma .
$$


The action (2.6) can now be written in the first order form

$$
\begin{aligned}
S & =\int d r \int d^{d} y \sqrt{\tilde{h}}\left(\pi^{i j}\left(\dot{\tilde{h}}_{i j}-2 N K_{i j}-\nabla_{i} N_{j}-\nabla_{j} N_{i}\right)+\pi_{\phi}\left(\dot{\phi}-N \Sigma-£_{N} \phi\right)+\mathscr{L}\right) \\
& =\int d r \int d^{d} y \sqrt{\tilde{h}}\left(\pi^{i j} \dot{\tilde{h}}_{i j}+\pi_{\phi} \dot{\phi}+P^{i j} \dot{K}_{i j}+P_{\Sigma} \dot{\Sigma}-\mathscr{H}\right),
\end{aligned}
$$

with

$$
\mathscr{H}=\pi^{i j}\left(2 N K_{i j}+\nabla_{i} N_{j}+\nabla_{j} N_{i}\right)+\pi_{\phi}\left(N \Sigma+£_{N} \phi\right)+P^{i j} \dot{K}_{i j}+P_{\Sigma} \dot{\Sigma}-\mathscr{L},
$$

which can be brought to the form

$$
\mathscr{H}=N \mathcal{H}\left(\tilde{g}, \phi, K ; \pi, \pi_{\phi}, P-P^{\phi}\right)+N^{i} \mathcal{P}_{i}\left(\tilde{g}, \phi, K ; \pi, \pi_{\phi}, P-P^{\phi}\right),
$$

for appropriate $\mathcal{H}$ and $\mathcal{P}$ that can be easily worked out. For this one needs to use (A.12) and (2.12).

Requiring that the variation of $S$ vanishes gives us Hamilton's equations and a constraint at the boundary, which can be satisfied by either Dirichlet or Neumann boundary conditions for the variables $\tilde{h}_{i j}, \phi, K_{i j}$ and $\Sigma$. In order to impose Dirichlet boundary conditions for $\tilde{h}_{i j}$ and $\phi$, and Neumann boundary conditions for $K_{i j}$ and $\Sigma$, the action $S$ needs to be modified appropriately. This can be done by means of a canonical transformation so that instead of $S$ we use

$$
\begin{aligned}
\widehat{S} & =S-\int d^{d+1} x £_{t}\left(\sqrt{\tilde{h}}\left(P^{i j} K_{i j}+P_{\Sigma} \Sigma\right)\right) \\
& =\int d r \int d^{d} y \sqrt{\tilde{h}}\left(\pi^{i j} \dot{\tilde{h}}_{i j}+\pi_{\phi} \dot{\phi}-K_{i j} \dot{P}^{i j}-\Sigma \dot{P}_{\Sigma}-N \widehat{\mathcal{H}}-N^{i} \widehat{\mathcal{P}}_{i}\right)
\end{aligned}
$$

with

$$
\begin{aligned}
\widehat{\mathcal{H}} & =\mathcal{H}+K\left(K^{i j} P_{i j}+\Sigma P_{\Sigma}\right), \\
\widehat{\mathcal{P}}_{i} & =\mathcal{P}_{i}-\nabla_{i}\left(K^{j k} P_{j k}+\Sigma P_{\Sigma}\right) .
\end{aligned}
$$

Now we can impose Dirichlet boundary conditions for $\tilde{h}_{i j}$ and $\phi$, and Neumann boundary conditions for $K_{i j}$ and $\Sigma$. As we observe $\widehat{S}$ in (2.19) does not contain derivatives of $N$ or $N^{i}$, and so these act as Lagrange multipliers enforcing the Hamiltonian and momentum constraints

$$
\widehat{\mathcal{H}}=0 \quad \text { and } \quad \widehat{\mathcal{P}}_{i}=0 .
$$

To proceed we need to obtain an action defined at the boundary. Let $\overline{\tilde{h}}_{i j}, \bar{\phi}, \bar{P}_{i j}$ and $\bar{P}_{\Sigma}$ be the solutions of $\delta \widehat{S}=0$ with the appropriate boundary conditions. Using these solutions in $\widehat{S}$ and defining $\overline{\tilde{h}}_{i j}\left(y, r=r_{0}\right) \equiv \tilde{h}_{i j}(y)$ etc., gives us the classical action $S_{c}\left[\tilde{h}(y), \phi(y), P(y)-P^{\phi}(y), P_{\Sigma}(y)\right]$, and we have

$$
\widehat{\mathcal{H}}_{c}\left(\tilde{h}, \phi, K ; \pi, \pi_{\phi}, P-P_{\phi}\right)=0 \quad \text { and } \quad \widehat{\mathcal{P}}_{c}\left(\tilde{h}, \phi, K ; \pi, \pi_{\phi}, P-P_{\phi}\right)=0 .
$$

The Hamiltonian and momentum constraints (2.22) can be recast as equations for the reduced classical action $S_{r}$ defined as

$$
S_{r}[\tilde{h}, \phi]=S_{c}[\tilde{h}, \phi, 0,0],
$$


where the Neumann boundary conditions for $K_{i j}$ and $\Sigma$ have been used in $S_{c}$. The conjugate momenta on the boundary (fixed $r=r_{0}$ ) are given by

$$
\left.\pi\right|^{i j}=-\frac{1}{\sqrt{\tilde{h}}} \frac{\delta S_{r}}{\delta \tilde{h}_{i j}},\left.\quad \pi\right|_{\phi}=-\frac{1}{\sqrt{\tilde{h}}} \frac{\delta S_{r}}{\delta \phi} .
$$

The constraints (2.22) can be recast as constraints on $S_{r}$ by using $S$ of (2.6). Starting with $\mathscr{L}_{0}^{\tilde{h}}+\mathscr{L}_{0}^{\phi}$, which we write in the form $\pi^{i j} \dot{\tilde{h}}_{i j}+\pi_{\phi} \dot{\phi}-\mathscr{H}_{0}^{\tilde{h}}-\mathscr{H}_{0}^{\phi}$, we can use (2.10) to determine

$$
\pi_{i j}=K_{i j}-K \tilde{h}_{i j}, \quad \pi_{\phi}=£_{n} \phi
$$

which give

$$
K_{i j}=\pi_{i j}-\frac{1}{d-1} \pi \tilde{h}_{i j}, \quad £_{n} \phi=\pi_{\phi}
$$

Then,

$\mathscr{H}_{0}^{\tilde{h}}=N\left(\pi^{i j} \pi_{i j}-\frac{1}{d-1} \pi^{2}-2 \Lambda+R\right)-2 N^{i} \nabla^{j} \pi_{i j}, \quad \mathscr{H}_{0}^{\phi}=\frac{1}{2} N\left(\pi_{\phi}^{2}-\partial^{i} \phi \partial_{i} \phi\right)+\pi_{\phi} £_{N} \phi$,

and with definitions like in (2.18) we obtain

$$
\begin{array}{ll}
\mathcal{H}_{0}^{\tilde{h}}=\pi^{i j} \pi_{i j}-\frac{1}{d-1} \pi^{2}-2 \Lambda+R, & \mathcal{H}_{0}^{\phi}=\frac{1}{2}\left(\pi_{\phi}^{2}-\partial^{i} \phi \partial_{i} \phi\right), \\
\mathcal{P}_{i}^{\tilde{h}}=-2 \nabla^{j} \pi_{i j}, & \mathcal{P}_{i}^{\phi}=\pi_{\phi} \partial_{i} \phi .
\end{array}
$$

The projection of these expressions onto the boundary is trivial, and amounts essentially to $\pi_{i j},\left.\pi_{\phi} \rightarrow \pi\right|_{i j},\left.\pi\right|_{\phi}$ of (2.24), thus translating (2.22) into constraints on the form of the reduced classical action $S_{r}$.

The relations in (2.26) can also be directly obtained by the equations

$$
\left\{\int d^{d} y^{\prime} \sqrt{\tilde{h}} \mathscr{H}_{0}, \tilde{h}_{i j}(y)\right\}=\dot{\tilde{h}}_{i j}(y), \quad\left\{\int d^{d} y^{\prime} \sqrt{\tilde{h}} \mathscr{H}_{0}, \phi(y)\right\}=\dot{\phi}(y),
$$

where $\mathscr{H}_{0}=\mathscr{H}_{0}^{\tilde{h}}+\mathscr{H}_{0}^{\phi}$ and

$$
\{F(q, p), G(q, p)\}=\frac{\partial F}{\partial p} \cdot \frac{\partial G}{\partial q}-\frac{\partial F}{\partial q} \cdot \frac{\partial G}{\partial p} .
$$

The advantage of this method is that it can also be used at higher order due to the theorem of [15]. More specifically, from

$$
\left\{\int d^{d} y^{\prime} \sqrt{\tilde{h}} \mathscr{H}_{0}, K_{i j}(y)\right\}=\dot{K}_{i j}(y), \quad\left\{\int d^{d} y^{\prime} \sqrt{\tilde{h}} \mathscr{H}_{0}, \Sigma(y)\right\}=\dot{\Sigma}(y),
$$

we find that in (2.6) we may use

$$
\begin{aligned}
L_{i j}= & -\frac{1}{2(d-1)^{2}}\left(2(d-1) \Lambda+(d-1) R+(d-1) \pi^{k l} \pi_{k l}-3 \pi^{2}\right) \tilde{h}_{i j}+R_{i j} \\
& -\frac{3}{d-1} \pi \pi_{i j}+2 \pi_{i}^{k} \pi_{k j}+\frac{1}{4(d-1)}\left(\partial^{k} \phi \partial_{k} \phi-\pi_{\phi}^{2}\right) \tilde{h}_{i j}-\frac{1}{2} \partial_{i} \phi \partial_{j} \phi,
\end{aligned}
$$

and

$$
£_{n} £_{n} \phi-£_{a} \phi=\frac{1}{d-1} \pi \pi_{\phi}-\nabla^{2} \phi .
$$

We notice that (2.33) is the ADM decomposition of the bulk equation $\nabla^{2} \phi=0$. As a result, the coefficient $g$ of (2.2) will not contribute to the Hamiltonian. With our results it 
is straightforward to compute

$$
\begin{aligned}
\mathcal{H}_{1}^{\tilde{h}}= & \alpha_{1} \pi_{j}^{i} \pi^{j}{ }_{k} \pi_{l}^{k} \pi_{i}^{l}+\alpha_{2} \pi \pi_{j}^{i} \pi^{j}{ }_{k} \pi_{i}^{k}+\alpha_{3}\left(\pi^{i j} \pi_{i j}\right)^{2}+\alpha_{4} \pi^{2} \pi^{i j} \pi_{i j}+\alpha_{5} \pi^{4} \\
& +\beta_{1} \Lambda \pi^{i j} \pi_{i j}+\beta_{2} \Lambda \pi^{2}+\beta_{3} R \pi^{i j} \pi_{i j}+\beta_{4} R \pi^{2}+\beta_{5} R^{i j} \pi_{i}^{k} \pi_{k j}+\beta_{6} R^{i j} \pi \pi_{i j}+\beta_{7} R^{i j k l} \pi_{i k} \pi_{j l} \\
& +\gamma_{1} \pi^{i j} \nabla_{j} \nabla_{k} \pi_{i}^{k}+\gamma_{2} \pi^{i j} \nabla_{i} \partial_{j} \pi+\gamma_{3} \pi^{i j} \nabla^{2} \pi_{i j}+\gamma_{4} \pi \nabla^{i} \nabla^{j} \pi_{i j}+\gamma_{5} \pi \nabla^{2} \pi \\
& +\delta_{1} \Lambda^{2}+\delta_{2} \Lambda R+\delta_{3} R^{2}+\delta_{4} R^{i j} R_{i j}+\delta_{5} R^{i j k l} R_{i j k l},
\end{aligned}
$$

and

$$
\begin{aligned}
\mathcal{H}_{1}^{\phi}= & \epsilon_{1} \pi^{i j} \pi_{i j} \pi_{\phi}^{2}+\epsilon_{2} \pi^{2} \pi_{\phi}^{2}+\epsilon_{3} \pi_{\phi}^{4} \\
& +\zeta_{1} \Lambda \pi_{\phi}^{2}+\zeta_{2} R \pi_{\phi}^{2} \\
& +\eta_{1} \pi^{i j} \pi_{\phi} \nabla_{i} \partial_{j} \phi+\eta_{2} \pi \pi_{\phi} \nabla^{2} \phi+\eta_{3} \pi_{k}^{i} \pi^{k j} \partial_{i} \phi \partial_{j} \phi+\eta_{4} \pi \pi^{i j} \partial_{i} \phi \partial_{j} \phi \\
& +\eta_{5} \pi^{i j} \pi_{i j} \partial^{k} \phi \partial_{k} \phi+\eta_{6} \pi^{2} \partial^{i} \phi \partial_{i} \phi+\eta_{7} \pi_{\phi}^{2} \partial^{i} \phi \partial_{i} \phi \\
& +\theta_{1} \partial^{i} \pi_{\phi} \partial_{i} \pi_{\phi}+\theta_{2} \pi^{i j} \partial_{i} \pi_{\phi} \partial_{j} \phi+\theta_{3} \pi \partial^{i} \pi_{\phi} \partial_{i} \phi \\
& +\kappa_{1} \Lambda \partial^{i} \phi \partial_{i} \phi+\kappa_{2} R \partial^{i} \phi \partial_{i} \phi+\kappa_{3} R^{i j} \partial_{i} \phi \partial_{j} \phi+\kappa_{4} \nabla^{2} \phi \nabla^{2} \phi+\kappa_{5} \nabla^{i} \partial^{j} \phi \nabla_{i} \partial_{j} \phi \\
& +\lambda\left(\partial^{i} \phi \partial_{i} \phi\right)^{2},
\end{aligned}
$$

with

$$
\begin{aligned}
& \alpha_{1}=2 c, \quad \alpha_{2}=\frac{2}{d-1} x_{5}, \\
& \alpha_{3}=\frac{1}{4(d-1)^{2}}\left(4 a+\left(d^{2}-3 d+4\right) b+4(d-2)(2 d-3) c-2(d-1)\left(d x_{4}+3 x_{5}\right)\right) \text {, } \\
& \alpha_{4}=-\frac{1}{2(d-1)^{3}}\left(4 a+\left(d^{2}-3 d+4\right) b+4\left(2 d^{2}-5 d+4\right) c\right. \\
& \left.+3 d x_{3}-\left(2 d^{2}-7 d+2\right) x_{4}+3(2 d-1) x_{5}\right), \\
& \alpha_{5}=\frac{1}{4(d-1)^{4}}\left(4 a+\left(d^{2}-3 d+4\right) b+4\left(2 d^{2}-5 d+4\right) c\right. \\
& \left.+2(3 d-4) x_{3}-2\left(d^{2}-6 d+6\right) x_{4}+2(5 d-6) x_{5}\right) \text {, } \\
& \beta_{1}=\frac{1}{(d-1)^{2}}\left(4 d a-d(d-3) b-4(d-2) c-(d-1)\left(d x_{4}+3 x_{5}\right)\right), \\
& \beta_{2}=-\frac{1}{(d-1)^{3}}\left(4 d a-d(d-3) b-4(d-2) c+3 d x_{3}-\left(d^{2}-2 d-2\right) x_{4}-3(d-2) x_{5}\right), \\
& \beta_{3}=\frac{1}{2(d-1)^{2}}\left(4 a+\left(d^{2}-3 d+4\right) b-4(3 d-4) c-(d-1)\left(d x_{1}+x_{2}-(d-2) x_{4}+3 x_{5}\right)\right), \\
& \beta_{4}=-\frac{1}{2(d-1)^{3}}\left(4 a+\left(d^{2}-3 d+4\right) b-4(d-2) c\right. \\
& \left.-(d-1)(d-4) x_{1}+3(d-1) x_{2}-3(d-2) x_{3}+\left(d^{2}-8 d+10\right) x_{4}-3(3 d-4) x_{5}\right), \\
& \beta_{5}=16 c+3 x_{5}, \quad \beta_{6}=\frac{2}{d-1}\left(x_{1}+2 x_{2}-x_{4}-3 x_{5}\right), \quad \beta_{7}=-2\left(6 c+x_{2}\right), \\
& \gamma_{1}=-2\left(b-4 c-x_{2}\right), \quad \gamma_{2}=-\frac{1}{d-1}\left(2 b+8 c+2 x_{1}+x_{2}\right), \quad \gamma_{3}=-8 c-x_{2}, \\
& \gamma_{4}=\frac{2}{d-1}\left(b-4 c-x_{2}\right), \quad \gamma_{5}=\frac{1}{d-1}\left(8 c+x_{2}\right),
\end{aligned}
$$




$$
\begin{aligned}
& \delta_{1}=\frac{d}{(d-1)^{2}}(4 d a+(d+1) b+4 c), \\
& \delta_{2}=\frac{1}{(d-1)^{2}}\left(4 d a-d(d-3) b-4(d-2) c-(d-1)\left(d x_{1}+x_{2}\right)\right), \\
& \delta_{3}=\frac{1}{4(d-1)^{2}}\left(4 a+\left(d^{2}-3 d+4\right) b-4(3 d-4) c+2(d-1)\left((d-2) x_{1}-x_{2}\right)\right), \\
& \delta_{4}=4 c+x_{2}, \quad \delta_{5}=c,
\end{aligned}
$$

and

$$
\begin{aligned}
& \epsilon_{1}=\frac{1}{4(d-1)^{2}}(4 d a-d(d-3) b-4(d-2) c-(d-1)(4 e-2(d-2) f+4(d-1) h) \\
& \left.-(d-1)\left(d x_{4}+3 x_{5}\right)\right), \\
& \epsilon_{2}=-\frac{1}{4(d-1)^{3}}(4 d a-d(d-3) b-4(d-2) c-2(d-1)(2 e-(d-2) f+2(d-3) h) \\
& \left.+3 d x_{3}-\left(d^{2}-2 d-2\right) x_{4}-3(d-2) x_{5}\right) \text {, } \\
& \epsilon_{3}=\frac{d}{16(d-1)^{2}}(4 d a+(d+1) b+4 c-4(d-1)(2 e+f)) \text {, } \\
& \zeta_{1}=\frac{d}{2(d-1)^{2}}(4 d a+(d+1) b+4 c-2(d-1)(2 e+f)) \\
& \zeta_{2}=\frac{1}{4(d-1)^{2}}\left(4 d a-d(d-3) b-4(d-2) c-2(d-1)(2 e-(d-2) f)-(d-1)\left(d x_{1}+x_{2}\right)\right), \\
& \eta_{1}=2(f-h), \quad \eta_{2}=\frac{1}{d-1}\left(4 h-y_{3}\right), \quad \eta_{3}=-\frac{1}{2}\left(8 c+4 h+3 x_{5}+4 y_{2}\right), \\
& \eta_{4}=\frac{1}{d-1}\left(4 c+4 h+x_{4}+3 x_{5}+2 y_{1}+4 y_{2}\right), \\
& \eta_{5}=\frac{1}{4(d-1)^{2}}(4(d-2) a-(d-2)(d-3) b+4(3 d-4) c-2(d-1)(2 e+f) \\
& \left.-(d-1)\left((d-2) x_{4}-3 x_{5}+2 d y_{1}+2 y_{2}\right)\right), \\
& \eta_{6}=-\frac{1}{4(d-1)^{3}}((d-2)(4 a-(d-3) b)+4(3 d-4) c-2(d-1)(2 e+f-4 h) \\
& \left.+3(d-2) x_{3}-\left(d^{2}-8 d+10\right) x_{4}+3(3 d-4) x_{5}-2(d-1)\left((d-4) y_{1}-3 y_{2}\right)\right), \\
& \eta_{7}=\frac{1}{8(d-1)^{2}}\left((d-2)(4 d a+(d+1) b+4 c)-2(d-1)\left((d-1)(4 e+f)+d y_{1}+y_{2}\right)\right) \text {, } \\
& \theta_{1}=-2 h+y_{3}, \quad \theta_{2}=2\left(f+2 h+y_{2}-y_{3}\right), \quad \theta_{3}=-\frac{1}{d-1}\left(4 h+2 y_{1}+2 y_{2}-y_{3}\right), \\
& \kappa_{1}=\frac{1}{2(d-1)^{2}}\left((d-2)(4 d a+(d+1) b+4 c)-2(d-1)\left(2 d e+f+d y_{1}+y_{2}\right)\right), \\
& \kappa_{2}=\frac{1}{4(d-1)^{2}}((d-2)(4 a-(d-3) b)+4(3 d-4) c-2(d-1)(2 e+f) \\
& \left.-(d-1)\left((d-2) x_{1}-x_{2}-2(d-2) y_{1}-2 y_{2}\right)\right), \\
& \kappa_{3}=-\frac{1}{2}\left(8 c+x_{2}-2 y_{2}\right), \quad \kappa_{4}=-h+y_{3}, \quad \kappa_{5}=-h, \\
& \lambda=\frac{1}{16(d-1)^{2}}\left(4(d-2)^{2} a+\left(5 d^{2}-15 d+12\right) b+4\left(4 d^{2}-11 d+8\right) c\right. \\
& \left.-4(d-1)\left(2(d-2) e+(2 d-3) f+(d-2) y_{1}+(2 d-3) y_{2}\right)\right) \text {. }
\end{aligned}
$$


As expected, $g$ does not appear in any of these constants. From now on we will only consider terms quadratic in $\phi$ so that $\lambda$ above will not be used.

With these results we can write down the equation that gives us the reduced classical action, namely

$$
\mathcal{H}_{r}\left(\tilde{h}, \phi, \pi|, \pi|_{\phi}\right) \equiv \mathcal{H}_{0}^{\tilde{h}}+\mathcal{H}_{0}^{\phi}+\mathcal{H}_{1}^{\tilde{h}}+\mathcal{H}_{1}^{\phi}=0 .
$$

This can be written as a flow equation

$$
\left\{S_{r}, S_{r}\right\}+\left\{S_{r}, S_{r}, S_{r}, S_{r}\right\}=\mathscr{L}_{d},
$$

where the two-bracket is given by

$$
\begin{aligned}
\tilde{h}\left\{S_{r}, S_{r}\right\}= & \left(1+\beta_{1} \Lambda+\beta_{3} R\right) \tilde{h}_{i k} \tilde{h}_{j l} \frac{\delta S_{r}}{\delta \tilde{h}_{i j}} \frac{\delta S_{r}}{\delta \tilde{h}_{k l}}-\left(\frac{1}{d-1}-\beta_{2} \Lambda-\beta_{4} R\right)\left(\tilde{h}_{i j} \frac{\delta S_{r}}{\delta \tilde{h}_{i j}}\right)^{2} \\
& +\beta_{5} R_{i k} \tilde{h}_{j l} \frac{\delta S_{r}}{\delta \tilde{h}_{i j}} \frac{\delta S_{r}}{\delta \tilde{h}_{k l}}+\beta_{6} R_{i j} \tilde{h}_{k l} \frac{\delta S_{r}}{\delta \tilde{h}_{i j}} \frac{\delta S_{r}}{\delta \tilde{h}_{k l}}+\beta_{7} R_{i j k l} \frac{\delta S_{r}}{\delta \tilde{h}_{i k}} \frac{\delta S_{r}}{\delta \tilde{h}_{j l}} \\
& +\gamma_{1} \tilde{h}_{i j} \frac{\delta S_{r}}{\delta \tilde{h}_{i k}} \nabla_{k} \nabla_{l} \frac{\delta S_{r}}{\delta \tilde{h}_{l j}}+\gamma_{2} \frac{\delta S_{r}}{\delta \tilde{h}_{k l}} \nabla_{k} \partial_{l}\left(\tilde{h}_{i j} \frac{\delta S_{r}}{\delta \tilde{h}_{i j}}\right)+\gamma_{3} \tilde{h}_{i k} \tilde{h}_{j l} \frac{\delta S_{r}}{\delta \tilde{h}_{i j}} \nabla^{2} \frac{\delta S_{r}}{\delta \tilde{h}_{k l}} \\
& +\gamma_{4} \tilde{h}_{i j} \frac{\delta S_{r}}{\delta \tilde{h}_{i j}} \nabla_{k} \nabla_{l} \frac{\delta S_{r}}{\delta \tilde{h}_{k l}}+\gamma_{5} \tilde{h}_{i j} \tilde{h}_{k l} \frac{\delta S_{r}}{\delta \tilde{h}_{i j}} \nabla^{2} \frac{\delta S_{r}}{\delta \tilde{h}_{k l}} \\
& +\left(\frac{1}{2}+\zeta_{1} \Lambda+\zeta_{2} R\right)\left(\frac{\delta S_{r}}{\delta \phi}\right)^{2} \\
& +\eta_{1} \frac{\delta S_{r}}{\delta \tilde{h}_{i j}} \frac{\delta S_{r}}{\delta \phi} \nabla^{i} \partial^{j} \phi+\eta_{2} \tilde{h}_{i j} \frac{\delta S_{r}}{\delta \tilde{h}_{i j}} \frac{\delta S_{r}}{\delta \phi} \nabla^{2} \phi+\eta_{3} \tilde{h}_{i k} \frac{\delta S_{r}}{\delta \tilde{h}_{i j}} \frac{\delta S_{r}}{\delta \tilde{h}_{k l}} \partial_{j} \phi \partial_{l} \phi \\
& +\eta_{4} \tilde{h}_{i j} \frac{\delta S_{r}}{\delta \tilde{h}_{i j}} \frac{\delta S_{r}}{\delta \tilde{h}_{k l}} \partial_{k} \phi \partial_{l} \phi+\eta_{5} \tilde{h}_{i k} \tilde{h}_{j l} \frac{\delta S_{r}}{\delta \tilde{h}_{i j}} \frac{\delta S_{r}}{\delta \tilde{h}_{k l}} \partial^{m} \phi \partial_{m} \phi \\
& +\eta_{6}\left(\tilde{h}_{i j} \frac{\delta S_{r}}{\delta \tilde{h}_{i j}}\right)^{2} \partial^{k} \phi \partial_{k} \phi+\eta_{7}\left(\frac{\delta S_{r}}{\delta \phi}\right)^{2} \partial^{i} \phi \partial_{i} \phi \\
& +\theta_{1} \partial^{i} \frac{\delta S_{r}}{\delta \phi} \partial_{i} \frac{\delta S_{r}}{\delta \phi}+\theta_{2} \frac{\delta S_{r}}{\delta \tilde{h}_{i j}} \partial_{i} \frac{\delta S_{r}}{\delta \phi} \partial_{j} \phi+\theta_{3} \tilde{h}_{i j} \frac{\delta S_{r}}{\delta \tilde{h}_{i j}} \partial^{k} \frac{\delta S_{r}}{\delta \phi} \partial_{k} \phi
\end{aligned}
$$

the four-bracket is given by

$$
\begin{aligned}
\tilde{h}^{2}\left\{S_{r}, S_{r}, S_{r}, S_{r}\right\}= & \alpha_{1} \tilde{h}_{i q} \tilde{h}_{j k} \tilde{h}_{l m} \tilde{h}_{n p} \frac{\delta S_{r}}{\delta \tilde{h}_{i j}} \frac{\delta S_{r}}{\delta \tilde{h}_{k l}} \frac{\delta S_{r}}{\delta \tilde{h}_{m n}} \frac{\delta S_{r}}{\delta \tilde{h}_{p q}} \\
& +\alpha_{2} \tilde{h}_{i j} \frac{\delta S_{r}}{\delta \tilde{h}_{i j}} \tilde{h}_{k q} \tilde{h}_{l m} \tilde{h}_{n p} \frac{\delta S_{r}}{\delta \tilde{h}_{k l}} \frac{\delta S_{r}}{\delta \tilde{h}_{m n}} \frac{\delta S_{r}}{\delta \tilde{h}_{p q}}+\alpha_{3}\left(\tilde{h}_{i k} \tilde{h}_{j l} \frac{\delta S_{r}}{\delta \tilde{h}_{i j}} \frac{\delta S_{r}}{\delta \tilde{h}_{k l}}\right)^{2} \\
& +\alpha_{4}\left(\tilde{h}_{i j} \frac{\delta S_{r}}{\delta \tilde{h}_{i j}}\right)^{2} \tilde{h}_{k m} \tilde{h}_{l n} \frac{\delta S_{r}}{\delta \tilde{h}_{k l}} \frac{\delta S_{r}}{\delta \tilde{h}_{m n}}+\alpha_{5}\left(\tilde{h}_{i j} \frac{\delta S_{r}}{\delta \tilde{h}_{i j}}\right)^{4} \\
& +\epsilon_{1} \tilde{h}_{i k} \tilde{h}_{j l} \frac{\delta S_{r}}{\delta \tilde{h}_{i j}} \frac{\delta S_{r}}{\delta \tilde{h}_{k l}}\left(\frac{\delta S_{r}}{\delta \phi}\right)^{2}+\epsilon_{2}\left(\tilde{h}_{i j} \frac{\delta S_{r}}{\delta \tilde{h}_{i j}}\right)^{2}\left(\frac{\delta S_{r}}{\delta \phi}\right)^{2}+\epsilon_{3}\left(\frac{\delta S_{r}}{\delta \phi}\right)^{4},
\end{aligned}
$$


and

$$
\begin{aligned}
\mathscr{L}_{d}= & 2 \Lambda-R-\delta_{1} \Lambda^{2}-\delta_{2} \Lambda R-\delta_{3} R^{3}-\delta_{4} R^{i j} R_{i j}-\delta_{5} R^{i j k l} R_{i j k l} \\
& +\frac{1}{2} \partial^{i} \phi \partial_{i} \phi-\kappa_{1} \Lambda \partial^{i} \phi \partial_{i} \phi-\kappa_{2} R \partial^{i} \phi \partial_{i} \phi-\kappa_{3} R^{i j} \partial_{i} \phi \partial_{j} \phi-\kappa_{4} \nabla^{2} \phi \nabla^{2} \phi-\kappa_{5} \nabla^{i} \partial^{j} \phi \nabla_{i} \partial_{j} \phi .
\end{aligned}
$$

\section{Trace anomaly from the flow equation}

The flow equation (2.39) becomes useful if we make an ansatz for $S_{r}$ following [7, 8]:

$$
\frac{1}{2 \tilde{\kappa}_{d+1}^{2}} S_{r}[\tilde{h}, \phi]=\frac{1}{2 \tilde{\kappa}_{d+1}^{2}} S_{\mathrm{loc}}[\tilde{h}, \phi]+\Gamma[\tilde{h}, \phi],
$$

where $2 \tilde{\kappa}_{d+1}^{2}=16 \pi G_{d+1}$ with $G_{d+1}$ the $(d+1)$-dimensional Newton constant, $\Gamma$ is the generating functional of the boundary field theory, and $S_{\text {loc }}$ contains local counterterms. Equation (3.1) is only valid close to the boundary. Contributions to $S_{\text {loc }}$ are classified according to their scaling behavior close to the boundary. This is described by an appropriately defined weight $w,{ }^{1}$ giving rise to the relation

$$
S_{\mathrm{loc}}=\int d^{d} y \sqrt{\tilde{h}} \mathscr{L}_{\mathrm{loc}}, \quad \mathscr{L}_{\mathrm{loc}}=\sum_{w=0,2, \ldots} \mathscr{L}_{\mathrm{loc}}^{(w)} .
$$

A derivative has $w=1$, and so a curvature has $w=2$. We now use (3.2) in (2.39) and obtain independent equations for every weight. For $w=0,2$ we find

$$
\begin{aligned}
& \left\{S_{\mathrm{loc}}, S_{\mathrm{loc}}\right\}_{w=0}+\left\{S_{\mathrm{loc}}, S_{\mathrm{loc}}, S_{\mathrm{loc}}, S_{\mathrm{loc}}\right\}_{w=0}=\left(2-\delta_{1} \Lambda\right) \Lambda, \\
& \left\{S_{\mathrm{loc}}, S_{\mathrm{loc}}\right\}_{w=2}+\left\{S_{\mathrm{loc}}, S_{\mathrm{loc}}, S_{\mathrm{loc}}, S_{\mathrm{loc}}\right\}_{w=2}=-\left(1+\delta_{2} \Lambda\right) R+\left(\frac{1}{2}-\kappa_{1} \Lambda\right) \partial^{i} \phi \partial_{i} \phi,
\end{aligned}
$$

respectively, which, with

$$
\Lambda=-\frac{d(d-1)}{2 \ell^{2}}+\frac{d(d-3)}{2 \ell^{4}}(d(d+1) a+d b+2 c),
$$

so that we have AdS space with radius $\ell$ asymptotically, allow us to determine

$$
\mathscr{L}_{\text {loc }}^{(0)}=W, \quad \mathscr{L}_{\text {loc }}^{(2)}=-\Phi R+\frac{1}{2} M \partial^{i} \phi \partial_{i} \phi,
$$

with

$$
\begin{aligned}
W= & -\frac{2(d-1)}{\ell}-\frac{1}{\ell^{3}}\left(4 d(d+1) a+4 d b+8 c+d\left(d^{2} x_{3}+d x_{4}+x_{5}\right)\right) \\
\Phi= & \frac{\ell}{d-2}-\frac{1}{\ell}\left(\frac{2}{(d-1)(d-2)}(d(d+1) a+d b+2 c)-d x_{1}-x_{2}-\frac{3}{2(d-1)}\left(d^{2} x_{3}+d x_{4}+x_{5}\right)\right), \\
M= & \frac{\ell}{d-2}+\frac{1}{\ell}\left(\frac{2}{d-1}(d(d+1) a+d b+2 c)-\frac{2}{d-2}(d(d+1) e+d f-2 h)\right. \\
& \left.+\frac{3}{2(d-1)}\left(d^{2} x_{3}+d x_{4}+x_{5}\right)-2\left(d y_{1}+y_{2}\right)\right),
\end{aligned}
$$

\footnotetext{
${ }^{1}$ See section 4 below for more details on the weight.
} 
where we choose the negative sign for the $1 / \ell$ term of $W$ so that the $\ell$ term of $M$ is positive. Note that we are only able to determine $W, \Phi$ and $M$ up to specific powers of $\ell$ as seen in (3.6), consistently with the terms included in (2.2) and (2.4). Further terms in the $\ell$-expansion of $W, \Phi$ and $M$ depend generally also on even higher-derivative terms than the ones considered in (2.2) and (2.4).

\subsection{Four-dimensional trace anomaly}

At weight four the four-dimensional trace anomaly can be evaluated using the definition

$$
\left\langle T_{i}^{i}\right\rangle=-\frac{2}{\sqrt{\tilde{h}}} \tilde{h}_{i j} \frac{\delta \Gamma}{\delta \tilde{h}_{i j}} .
$$

We assign weight four to $\delta \Gamma / \delta \tilde{h}_{i j}$ and $\delta \Gamma / \delta \phi$, and then (2.39) gives ${ }^{2}$

$$
\begin{aligned}
2\left\{S_{\mathrm{loc}}, \Gamma\right\}_{w=4}+4\left\{S_{\mathrm{loc}}, S_{\mathrm{loc}}, S_{\mathrm{loc}}, \Gamma\right\}_{w=4}= & -\frac{1}{2 \kappa_{5}^{2}}\left(\left\{S_{\mathrm{loc}}, S_{\mathrm{loc}}\right\}_{w=4}+\left\{S_{\mathrm{loc}}, S_{\mathrm{loc}}, S_{\mathrm{loc}}, S_{\mathrm{loc}}\right\}_{w=4}\right. \\
& +\delta_{3} R^{2}+\delta_{4} R^{i j} R_{i j}+\delta_{5} R^{i j k l} R_{i j k l} \\
& +\kappa_{2} R \partial^{i} \phi \partial_{i} \phi+\kappa_{3} R^{i j} \partial_{i} \phi \partial_{j} \phi \\
& \left.+\kappa_{4} \nabla^{2} \phi \nabla^{2} \phi+\kappa_{5} \nabla^{i} \partial^{j} \phi \nabla_{i} \partial_{j} \phi\right) .
\end{aligned}
$$

It turns out that with $W$ as in (3.6) the left-hand side of (3.8) does not contain a $1 / \ell^{3}$ contribution for any $d$, and so it is simply equal to $(1 / \ell)\left\langle T_{i}^{i}\right\rangle$ at the order we're working in. It is also straightforward to work out the right-hand side of (3.8), and using (3.6) we finally find

$$
\begin{aligned}
\left\langle T_{i}^{i}\right\rangle= & \frac{\ell}{2 \tilde{\kappa}_{5}^{2}}\left(-\frac{1}{2}\left(\frac{1}{4} \ell^{2}-10 a-2 b-c\right) E_{4}+\frac{1}{2}\left(\frac{1}{4} \ell^{2}-10 a-2 b+c\right) W^{i j k l} W_{i j k l}\right. \\
& \left.+\frac{1}{2}\left(\frac{1}{4} \ell^{2}-10 e-2 f+2 h\right)\left(\nabla^{2} \phi \nabla^{2} \phi-2 R^{i j} \partial_{i} \phi \partial_{j} \phi+\frac{2}{3} R \partial^{i} \phi \partial_{i} \phi\right)\right),
\end{aligned}
$$

where the Euler term is

$$
E_{4}=R^{i j k l} R_{i j k l}-4 R^{i j} R_{i j}+R^{2},
$$

and the Weyl tensor is here the $d=4$ version of

$$
W_{i j k l}=R_{i j k l}+\frac{2}{d-2}\left(\tilde{h}_{i[l} R_{k] j}+\tilde{h}_{j[k} R_{l] i}\right)+\frac{2}{(d-1)(d-2)} \tilde{h}_{i[k} \tilde{h}_{l] j} R .
$$

In (3.9) the part of the anomaly quadratic in $\phi$ is in accord with the $d=4$ version of the Paneitz operator [19] (see also [20-23]). Note that although present throughout the calculation the constants $x_{1}, \ldots, x_{5}, y_{1}, y_{2}, y_{3}$ do not contribute to the final result (3.9).

\subsection{Six-dimensional trace anomaly}

To compute the $d=6$ anomaly we have to consider the weight-six part of the flow equation (2.39). At weight four we set

$$
\mathscr{L}_{\text {loc }}^{(4)}=X R^{2}+Y R^{i j} R_{i j}+Z R^{i j k l} R_{i j k l}+V R \partial^{i} \phi \partial_{i} \phi+U R^{i j} \partial_{i} \phi \partial_{j} \phi+T \nabla^{2} \phi \nabla^{2} \phi,
$$

\footnotetext{
${ }^{2}$ In (3.8) by $2\left\{S_{\mathrm{loc}}, \Gamma\right\}$ we mean $\left\{S_{\mathrm{loc}}, \Gamma\right\}+\left\{\Gamma, S_{\mathrm{loc}}\right\}$ and similarly for $4\left\{S_{\mathrm{loc}}, S_{\mathrm{loc}}, S_{\mathrm{loc}}, \Gamma\right\}$.
} 
and the weight-four part of (2.39) allows us to determine

$$
\begin{aligned}
& X=\frac{d \ell^{3}}{4(d-1)(d-2)^{2}(d-4)}-\frac{\ell}{2(d-1)^{2}(d-2)^{2}(d-4)}\left(3 d^{2}(d+1) a-3 d^{2} b+2\left(2 d^{2}-3 d+4\right) c\right) \\
& -\frac{\ell}{2(d-1)}\left(x_{1}-\frac{1}{d-2} x_{2}+\frac{1}{4(d-1)(d-2)^{2}}\left(3 d\left(d^{2}-8 d+8\right) x_{3}\right.\right. \\
& \left.\left.-\left(5 d^{2}+8 d-16\right) x_{4}-3(7 d-8) x_{5}\right)\right) \text {, } \\
& Y=-\frac{\ell^{3}}{(d-2)^{2}(d-4)}+\frac{2 \ell}{(d-1)(d-2)^{2}(d-4)}\left(3 d(d+1) a+3 d b+2\left(d^{2}-3 d+5\right) c\right) \\
& -\frac{\ell}{d-2}\left(x_{2}+\frac{1}{2(d-1)(d-2)}\left(3 d^{2} x_{3}+d(2 d+1) x_{4}+3(2 d+1) x_{5}\right)\right) \text {, } \\
& Z=-\frac{\ell}{d-4} c \text {, } \\
& V=-\frac{d \ell^{3}}{4(d-1)(d-2)^{2}(d-4)}-\frac{d \ell}{2(d-1)^{2}(d-2)^{2}}(d(d+1) a+d b+2 c) \\
& +\frac{\ell}{2(d-1)(d-2)^{2}(d-4)}\left(d^{2}(d+1) e+d^{2} f-2(3 d-4) h\right) \\
& +\frac{\ell}{4(d-1)}\left(x_{1}-\frac{1}{d-2} x_{2}+\frac{1}{2(d-1)(d-2)^{2}}\left(3 d\left(d^{2}-8 d+8\right) x_{3}\right.\right. \\
& \left.\left.-\left(5 d^{2}+8 d-16\right) x_{4}-3(7 d-8) x_{5}\right)\right)-\frac{\ell}{2(d-1)}\left(y_{1}-\frac{1}{d-2} y_{2}\right), \\
& U=\frac{\ell^{3}}{(d-2)^{2}(d-4)}+\frac{2 \ell}{(d-1)(d-2)^{2}}(d(d+1) a+d b+2 c) \\
& -\frac{\ell}{(d-2)^{2}(d-4)}\left(2 d(d+1) e+2 d f+\left(d^{2}-8 d+8\right) h\right) \\
& +\frac{\ell}{2(d-2)}\left(x_{2}+\frac{1}{(d-1)(d-2)}\left(3 d^{2} x_{3}+d(2 d+1) x_{4}+3(2 d-1) x_{5}\right)\right)-\frac{\ell}{d-2} y_{2}, \\
& T=-\frac{\ell^{3}}{2(d-2)^{2}(d-4)}-\frac{\ell}{(d-1)(d-2)^{2}}(d(d+1) a+d b+2 c) \\
& +\frac{\ell}{(d-2)^{2}(d-4)}\left(d(d+1) e+d f+d^{2} g+\left(d^{2}-9 d+16\right) h\right) \\
& -\frac{3 \ell}{4(d-1)(d-2)^{2}}\left(d^{2} x_{3}+d x_{4}+x_{5}\right)-\frac{\ell}{d-2} y_{3} .
\end{aligned}
$$

With these results as well as (3.6) and the weight-six part of (2.39) we can finally get the holographic trace anomaly in $d=6$ :

$$
\begin{aligned}
\left\langle T_{i}^{i}\right\rangle= & \frac{\ell^{3}}{2 \tilde{\kappa}_{7}^{2}}\left(\frac{1}{48}\left(\frac{1}{4} \ell^{2}-21 a-3 b-c\right) E_{6}-\frac{1}{4}\left(\frac{1}{4} \ell^{2}-21 a-3 b+\frac{1}{3} c\right) I_{1}\right. \\
& -\frac{1}{16}\left(\frac{1}{4} \ell^{2}-21 a-3 b-\frac{7}{3} c\right) I_{2}+\frac{1}{48}\left(\frac{1}{4} \ell^{2}-21 a-3 b+3 c\right) I_{3} \\
& +\frac{1}{48}\left(\frac{1}{4} \ell^{2}-21 a-3 b+3 c\right) J_{1}+\frac{1}{6}\left(\frac{1}{4} \ell^{2}-21 a-3 b+3 c\right) J_{2} \\
& -\frac{1}{8}\left(\frac{1}{4} \ell^{2}-21 a-3 b+3 c\right) J_{3}+\frac{1}{48}\left(\frac{1}{4} \ell^{2}-21 a-3 b+3 c\right) J_{4}
\end{aligned}
$$




$$
\begin{aligned}
& -\frac{1}{32}\left(\frac{1}{4} \ell^{2}-21 e-3 f+3 h\right)\left(\partial^{i} \nabla^{2} \phi \partial_{i} \nabla^{2} \phi-4 R^{i j} \nabla_{i} \partial_{j} \phi \nabla^{2} \phi+R \nabla^{2} \phi \nabla^{2} \phi\right. \\
& +2\left(2 R^{i k j l} R_{k l}+R^{i k} R_{k}^{j}-R R^{i j}+\nabla^{2} R^{i j}\right) \partial_{i} \phi \partial_{j} \phi \\
& \left.-\left(R^{i j} R_{i j}-\frac{9}{25} R^{2}+\frac{3}{5} \nabla^{2} R\right) \partial^{k} \phi \partial_{k} \phi\right) \\
& \left.-\frac{1}{4} c W^{i k l m} W_{k l m}^{j} \partial_{i} \phi \partial_{j} \phi+\frac{1}{20} c W^{i j k l} W_{i j k l} \partial^{m} \phi \partial_{m} \phi\right) .
\end{aligned}
$$

Here $E_{6}$ is the Euler term in six dimensions and $I_{1,2,3}$ the three terms with Weyl-invariant densities. Explicit expressions for these, as well as for the trivial anomalies $J_{1, \ldots, 4}$, are given in appendix $\mathrm{C}$. The first three lines in the part quadratic in $\phi$ are in accord with the Branson operator [26], while the next two terms involving the Weyl tensor were shown to appear generally in CFTs in six dimensions in [27]. Note that total derivatives have been dropped in (3.14). Just like in (3.9) the constants $x_{1}, \ldots, x_{5}, y_{1}, y_{2}, y_{3}$ do not contribute to the final result (3.14).

We note here that the results (3.9) and (3.14) have an obvious generalization to the case where $\phi \rightarrow \phi^{a}$, where $a$ is a flavor index.

\subsection{Bounds}

From the field theory point of view there are bounds derived by requiring positivity of the energy flux in lightlike directions [28]. These are bounds on the three-point function of the stress-energy tensor, which in $d=6$ take the form [30]

$$
\begin{aligned}
C_{1} & \equiv 1-\frac{1}{5} t_{2}-\frac{2}{35} t_{4} \geq 0, \quad C_{2} \equiv 1-\frac{1}{5} t_{2}-\frac{2}{35} t_{4}+\frac{1}{2} t_{2} \geq 0, \\
C_{3} & \equiv 1-\frac{1}{5} t_{2}-\frac{2}{35} t_{4}+\frac{4}{5}\left(t_{2}+t_{4}\right),
\end{aligned}
$$

where $t_{2}$ and $t_{4}$ correspond to the angular dependencies of the energy flux at null infinity. They are related to the coefficients $c_{1}, c_{2}$ and $c_{3}$ of the two- and three-point function of the stress-energy tensor by

$$
t_{2}=\frac{15\left(23 c_{1}-44 c_{2}+144 c_{3}\right)}{16 c_{3}}, \quad t_{4}=-\frac{105\left(c_{1}-2 c_{2}+6 c_{3}\right)}{2 c_{3}},
$$

where we use results for free fields first obtained in [46] (see also [27, 30]). The coefficient $c_{3}$ appears in the two-point function of the stress-energy tensor and thus $c_{3}>0$.

Our computation (3.14) allows us to determine

$$
\begin{aligned}
& c_{1}=-\frac{1}{4800 \sqrt{10}} \frac{L^{5}}{2 \tilde{\kappa}_{7}^{2}}(5+\sqrt{25-60 z})^{3 / 2}(3(5-20 z+\sqrt{25-60 z})+160 \tilde{c}), \\
& c_{2}=-\frac{1}{19200 \sqrt{10}} \frac{L^{5}}{2 \tilde{\kappa}_{7}^{2}}(5+\sqrt{25-60 z})^{3 / 2}(3(5-20 z+\sqrt{25-60 z})-160 \tilde{c}), \\
& c_{3}=\frac{1}{19200 \sqrt{10}} \frac{L^{5}}{2 \tilde{\kappa}_{7}^{2}}(5+\sqrt{25-60 z})^{3 / 2}(5-20 z+\sqrt{25-60 z}+160 \tilde{c})
\end{aligned}
$$


where

$$
L^{2}=-15 / \Lambda, \quad z=42 \tilde{a}+6 \tilde{b}+2 \tilde{c}, \quad \tilde{a}=a / L^{2}, \quad \tilde{b}=b / L^{2}, \quad \tilde{c}=c / L^{2} .
$$

We note that (3.17) are invariant under field redefinitions. ${ }^{3}$ With these results we can now use (3.15) with (3.16) to obtain

$$
-\frac{1}{880}(5-20 z+\sqrt{25-60 z}) \leq \tilde{c} \leq \frac{1}{80}(5-20 z+\sqrt{25-60 z}), \quad z \leq \frac{7}{20} .
$$

As we see $\tilde{c}$ can take both negative and positive values. For Gauss-Bonnet gravity in the bulk we reproduce the result of [30-32] ( $\tilde{c} \rightarrow \lambda / 12$ in their notation),

$$
-\frac{5}{192} \leq \tilde{c} \leq \frac{1}{64}
$$

\section{Trace anomaly away from fixed points}

In this section we establish a connection with Osborn's local RG. We will work in Einstein gravity with scalar fields $\phi^{a}$. Since we are now interested in the flow of the boundary theory we will keep the $\phi$-dependence of the various quantities that enter our expressions. Our flow equation is now

$$
\left\{S_{r}, S_{r}\right\}=\mathscr{L}_{d}
$$

with

$$
\begin{aligned}
\tilde{h}\left\{S_{r}, S_{r}\right\} & =\tilde{h}_{i k} \tilde{h}_{j l} \frac{\delta S_{r}}{\delta \tilde{h}_{i j}} \frac{\delta S_{r}}{\delta \tilde{h}_{k l}}-\frac{1}{d-1}\left(\tilde{h}_{i j} \frac{\delta S_{r}}{\delta \tilde{h}_{i j}}\right)^{2}+\frac{1}{2} H^{a b}(\phi) \frac{\delta S_{r}}{\delta \phi^{a}} \frac{\delta S_{r}}{\delta \phi^{b}}, \\
\mathscr{L}_{d} & =V(\phi)-R+\frac{1}{2} H_{a b}(\phi) \partial^{i} \phi^{a} \partial_{i} \phi^{b} .
\end{aligned}
$$

Following the prescription of [7], we use the splitting (3.1) and write down the local terms up to second order in derivatives:

$$
\mathscr{L}_{\mathrm{loc}}=W(\phi)-\Phi(\phi) R+\frac{1}{2} M_{a b}(\phi) \partial^{i} \phi^{a} \partial_{i} \phi^{b} .
$$

Using (4.3) we collect terms of the same functional form in (4.1) and find

$$
\begin{aligned}
V & =\frac{1}{2} H^{a b} \partial_{a} W \partial_{b} W-\frac{d}{4(d-1)} W^{2}, \\
-1 & =\frac{d-2}{2(d-1)} W \Phi-H^{a b} \partial_{a} W \partial_{b} \Phi, \\
\frac{1}{2} H_{a b} & =-\frac{d-2}{4(d-1)} W M_{a b}-W \partial_{a} \partial_{b} \Phi-\Gamma_{a b c}^{M} \partial_{d} W H^{c d}, \\
0 & =W \partial_{a} \Phi+M_{a b} \partial_{c} W H^{b c},
\end{aligned}
$$

\footnotetext{
${ }^{3}$ With Einstein or Lovelock gravity in the bulk $c_{1}, c_{2}$ and $c_{3}$ in (3.17) are such that $t_{4}=0$. This implies that the corresponding boundary theory is superconformal [30].
} 
where $\Gamma_{a b c}^{M}=\frac{1}{2}\left(\partial_{a} M_{b c}+\partial_{b} M_{a c}-\partial_{c} M_{a b}\right)$ and $\partial_{a}=\partial / \partial \phi^{a}$. The holographic beta function is given by

$$
\beta^{a}=-2(d-1) H^{a b} \partial_{b} \log W
$$

where, although $W$ is negative ${ }^{4}$ and dimensionful, we use $\partial_{a} \log W$ for $\partial_{a} W / W$.

In order to establish a connection with Osborn's local RG, we have to study the scaling behavior of the scalar fields $\phi$, which are viewed as sources in the dual field theory. This is done by introducing a mass term in the bulk potential. For convenience we use $\phi$ for the massless bulk scalar fields, and $\chi$ for the massive ones with mass $m_{\chi}$. By solving (4.4a) perturbatively to second order in $\chi$, one can obtain $\Delta_{\chi}=\frac{1}{2} d+\sqrt{\frac{1}{4} d^{2}+m_{\chi}^{2} \ell^{2}}[7]$. This reproduces the standard relation between the mass $m_{\chi}$ of the scalar field $\chi$ and the scaling dimension $\Delta_{\chi}$ of the dual operator. To obtain the trace anomaly from the flow equation (4.1), we have to assign "weight" zero to the scalar fields $\phi$, and weight $d-\Delta_{\chi}$ to $\chi$. This can be shown to be equivalent to the prescription of [8], which uses a scaling argument.

\subsection{Marginal operators}

Here we only include massless scalar fieds $\phi$, which correspond to marginal operators in the dual field theory. In this case, $S_{\text {loc }}$ nicely breaks down to separate contributions of weight zero and two, with

$$
\mathscr{L}_{\mathrm{loc}}^{(0)}=W(\phi), \quad \mathscr{L}_{\mathrm{loc}}^{(2)}=-\Phi(\phi) R+\frac{1}{2} M_{a b}(\phi) \partial^{i} \phi^{a} \partial_{i} \phi^{b}
$$

The weight- $d$ part of the flow equation (4.1) can be brought to the form

$$
-\frac{2}{\sqrt{\tilde{h}}} \tilde{h}_{i j} \frac{\delta \Gamma}{\delta \tilde{h}_{i j}}-\beta^{a} \frac{1}{\sqrt{\tilde{h}}} \frac{\delta \Gamma}{\delta \phi^{a}}=-\frac{1}{2 \tilde{\kappa}_{d+1}^{2}} \frac{2(d-1)}{W}\left\{S_{\mathrm{loc}}, S_{\mathrm{loc}}\right\}_{w=d} .
$$

The left-hand side of (4.7) is clearly $\left\langle T^{i}{ }_{i}\right\rangle-\beta^{a}\left\langle\mathcal{O}_{a}\right\rangle$, where $\mathcal{O}_{a}$ are marginal operators. The right-hand side of (4.7) can be easily computed using (4.2). It is clear that (4.7) is the holographic counterpart of the local RG equation of Osborn [33]. We should note here that (4.7) involves bare quantities, but as was shown already in [7] we can essentially write down the same equation with the renormalized quantities.

Osborn's expression is the starting point for the derivation of Weyl consistency conditions that include an equation that resembles an $a$-theorem. Here we will compute the various quantities that enter Osborn's expression holographically, focusing in the four-

\footnotetext{
${ }^{4}$ We choose $W<0$ so that $H_{a b}$ and $M_{a b}$ can be taken positive-definite consistently with (4.4c) in the limit where the $\phi$ dependence is neglected. In that case $\Phi$ is positive as can be seen from (4.4b).
} 
dimensional case. Using (4.2) we compute

$$
\begin{aligned}
\left\{S_{\mathrm{loc}}, S_{\mathrm{loc}}\right\}_{w=4}= & -\frac{1}{2} \Phi^{2} E_{4}+\frac{1}{2} \Phi^{2} W^{i j k l} W_{i j k l}+\frac{1}{2} H^{a b} \partial_{a} \Phi \partial_{b} \Phi R^{2} \\
& -2 \Phi \partial_{a} \Phi G^{i j} \nabla_{i} \partial_{j} \phi^{a}+M_{a b} \partial_{c} \Phi H^{b c} R \nabla^{2} \phi^{a} \\
& -\Phi\left(M_{a b}+2 \partial_{a} \partial_{b} \Phi\right) G^{i j} \partial_{i} \phi^{a} \partial_{j} \phi^{b}-\frac{1}{6}\left(\Phi M_{a b}-6 \Gamma_{a b c}^{M} \partial_{d} \Phi H^{c d}\right) R \partial^{i} \phi^{a} \partial_{i} \phi^{b} \\
& +\frac{1}{2}\left(M_{a c} M_{b d} H^{c d}-2 \partial_{a} \Phi \partial_{b} \Phi\right) \nabla^{2} \phi^{a} \nabla^{2} \phi^{b}+\partial_{a} \Phi \partial_{b} \Phi \nabla^{i} \partial^{j} \phi^{a} \nabla_{i} \partial_{j} \phi^{b} \\
& -\frac{1}{2}\left(M_{a b} \partial_{c} \Phi+4 \partial_{a} \partial_{b} \Phi \partial_{c} \Phi-2 \Gamma_{a b d}^{M} M_{c e} H^{d e}\right) \partial^{i} \phi^{a} \partial_{i} \phi^{b} \nabla^{2} \phi^{c} \\
& +\left(M_{a b} \partial_{c} \Phi+2 \partial_{a} \partial_{b} \Phi \partial_{c} \Phi\right) \partial^{i} \phi^{a} \partial^{j} \phi^{b} \nabla_{i} \partial_{j} \phi^{c} \\
& -\left(\frac{1}{12} M_{a b} M_{c d}-\frac{1}{4} M_{a c} M_{b d}+\frac{1}{2} \Gamma_{a b e}^{M} \Gamma_{c d f}^{M} H^{e f}\right. \\
& \left.+\frac{1}{2} M_{a b} \partial_{c} \partial_{d} \Phi-M_{a c} \partial_{b} \partial_{d} \Phi+\partial_{a} \partial_{b} \Phi \partial_{c} \partial_{d} \Phi-\partial_{a} \partial_{c} \Phi \partial_{b} \partial_{d} \Phi\right) \partial^{i} \phi^{a} \partial_{i} \phi^{b} \partial^{j} \phi^{c} \partial_{j} \phi^{d},
\end{aligned}
$$

where $G_{i j}$ is the Einstein tensor.

To match with Osborn's expression we write

$$
\left(\Delta_{\sigma}^{\mathrm{W}}-\Delta_{\sigma}^{\beta}\right) \Gamma=-\int d^{4} y \sqrt{\tilde{h}} \sigma \frac{1}{2 \tilde{\kappa}_{5}^{2}} \frac{6}{W}\left\{S_{\mathrm{loc}}, S_{\mathrm{loc}}\right\}_{w=4},
$$

where, with the definitions

$$
\Delta_{\sigma}^{\mathrm{W}}=-2 \int d^{4} y \sigma \tilde{h}_{i j} \frac{\delta}{\delta \tilde{h}_{i j}}, \quad \Delta_{\sigma}^{\beta}=\int d^{4} y \sigma \beta^{a} \frac{\delta}{\delta \phi^{a}},
$$

we have

$$
\Delta_{\sigma}^{\mathrm{W}} \Gamma=\int d^{4} y \sqrt{\tilde{h}} \sigma\left\langle T_{i}^{i}\right\rangle, \quad \Delta_{\sigma}^{\beta} \Gamma=\int d^{4} y \sqrt{\tilde{h}} \sigma \beta^{a}\left\langle\mathcal{O}_{a}\right\rangle .
$$

The general form of the anomaly is

$$
\begin{aligned}
\left(\Delta_{\sigma}^{\mathrm{W}}-\Delta_{\sigma}^{\beta}\right) \Gamma= & -\int d^{4} y \sqrt{\tilde{h}} \sigma\left(A E_{4}+B R^{2}-C W^{i j k l} W_{i j k l}\right. \\
& +\frac{1}{3} E_{a}^{\phi} \partial^{i} \phi^{a} \partial_{i} R+\frac{1}{6} F_{a b}^{\phi} \partial^{i} \phi^{a} \partial_{i} \phi^{b} R+\frac{1}{2} G_{a b}^{\phi} \partial_{i} \phi^{a} \partial_{j} \phi^{b} G^{i j} \\
& \left.+\frac{1}{2} A_{a b}^{\phi} \nabla^{2} \phi^{a} \nabla^{2} \phi^{b}+\frac{1}{2} B_{a b c}^{\phi} \partial^{i} \phi^{a} \partial_{i} \phi^{b} \nabla^{2} \phi^{c}+\frac{1}{4} C_{a b c d}^{\phi} \partial^{i} \phi^{a} \partial_{i} \phi^{b} \partial^{j} \phi^{c} \partial_{j} \phi^{d}\right) \\
& -\int d^{4} y \sqrt{\tilde{h}} \partial_{i} \sigma\left(W_{a}^{\phi} \partial_{j} \phi^{a} G^{i j}+\frac{1}{3} \partial^{i}(D R)+\frac{1}{3} Y_{a}^{\phi} \partial^{i} \phi R\right. \\
& \left.+\partial^{i}\left(U_{a}^{\phi} \nabla^{2} \phi^{a}+\frac{1}{2} V_{a b}^{\phi} \partial^{j} \phi^{a} \partial_{j} \phi^{b}\right)+S_{a b}^{\phi} \partial^{i} \phi^{a} \nabla^{2} \phi^{b}+\frac{1}{2} T_{a b c}^{\phi} \partial^{j} \phi^{a} \partial_{j} \phi^{b} \partial^{i} \phi^{c}\right),
\end{aligned}
$$

and it is now straightforward to match coefficients between (4.9) and (4.12) ${ }^{5}$ For example, we find

$$
A=C=-\frac{1}{2 \tilde{\kappa}_{5}^{2}} \frac{3}{W} \Phi^{2} .
$$

\footnotetext{
${ }^{5}$ The results can be found in appendix D.
} 
The fact that $A=C$ is a consequence of using only Einstein gravity in the bulk. Since $W<0$ we have $A, C>0$. We also have

$$
W_{a}^{\phi}=\frac{1}{2 \tilde{\kappa}_{5}^{2}} \frac{12}{W} \Phi \partial_{a} \Phi, \quad G_{a b}^{\phi}=-\frac{1}{2 \tilde{\kappa}_{5}^{2}} \frac{12}{W}\left(\left(M_{a b}+\partial_{a} \log W \partial_{b} \Phi+\partial_{b} \log W \partial_{a} \Phi\right) \Phi-\partial_{a} \Phi \partial_{b} \Phi\right),
$$

and we find that the consistency condition

$$
\partial_{a} \tilde{A}=\frac{1}{8}\left(G_{a b}^{\phi}+\partial_{a} W_{b}^{\phi}-\partial_{b} W_{a}^{\phi}\right) \beta^{b}, \quad \tilde{A}=A+\frac{1}{8} W_{a}^{\phi} \beta^{a},
$$

is satisfied with the use of $(4.4 \mathrm{~b})$ and $(4.4 \mathrm{~d}){ }^{6}{ }^{6}$ We also find that when the $\phi$-dependence of the various quantities is neglected, then $G_{a b}^{\phi}$ is positive-definite due to (4.4b) and (4.4c) if we take $W<0$ and $H_{a b}$ to be positive-definite. This gives results discussed in [41, 47], although the connection to these papers if the $\phi$-dependence is maintained is not clear. Positivity of $G_{a b}^{\phi}$ in perturbative field theory has been established in [34].

We also have

$$
A_{a b}^{\phi}=\frac{1}{2 \widetilde{\kappa}_{5}^{2}} \frac{6}{W} M_{a c} M_{b d} H^{c d},
$$

which is negative-definite as expected from the field-theoretic analysis [34]. If the $\phi$ dependence in (4.14) is neglected then we see using (3.6) that $G_{a b}^{\phi}=-2 A_{a b}^{\phi}$, a relation valid in conformal perturbation theory in field theory [34].

In $d=6$ one should be able to repeat the analysis above and check holographically the consistency conditions of [38]. Here we make a comment related to the metric analogous to $G_{a b}^{\phi}$ in $d=6, G_{6 a b}^{\phi}$. In [40] it was shown in multiflavor $\phi^{3}$ theory in $d=6$ that this metric is perturbatively negative-definite around the trivial fixed point. Furthermore, in [27] it was pointed out that this metric is proportional to the coefficient of the contribution $W^{i k l m} W^{j} k l m \partial_{i} \phi^{a} \partial_{j} \phi^{b}$ in the notation of (3.14). Using our result (3.14) we find

$$
G_{6 a b}^{\phi}=-\frac{1}{160 \sqrt{10}} \frac{L^{5}}{2 \tilde{\kappa}_{7}^{2}}(5+\sqrt{25-60 z})^{3 / 2} \tilde{c} \delta_{a b},
$$

at the fixed point, where $\delta_{a b}$ is the Kronecker delta and we use the definitions (3.18). If $\tilde{c}<0$ this would give us a positive-definite $G_{6 a b}^{\phi}$. However, our bound (3.19) shows that $\tilde{c}$ has an undetermined sign, and so we cannot determine the sign of $G_{6 a b}^{\phi}$ from (4.17) at the fixed point.

Besides the energy-positivity bounds discussed in section 3.3 there are more stringent constraints arising from causality considerations in the bulk [48]. More specifically, causality violations occur in the bulk for $c \neq 0$, unless there is an infinite tower of massive higher-spin fields. The significance of this result for the $a$-theorem in a six-dimensional field theory with nonvanishing $G_{6 a b}^{\phi}$ as in (4.17) is unclear.

\subsection{Relevant operators}

In order to include relevant scalar deformations we add scalar fields $\chi^{\alpha}$ with nonzero mass $m_{\chi}$. Then, the bulk Lagrangian becomes

$$
\mathscr{L}_{B}^{\tilde{g}, \phi, \chi}=V(\phi, \chi)-R+\frac{1}{2} H_{a b} \partial_{\mu} \phi^{a} \partial^{\mu} \phi^{b}+H_{\alpha a} \partial^{\mu} \chi^{\alpha} \partial_{\mu} \phi^{a}+\frac{1}{2} H_{\alpha \beta} \partial_{\mu} \chi^{\alpha} \partial^{\mu} \chi^{\beta} .
$$

\footnotetext{
${ }^{6}$ The remaining consistency conditions of [33] are also satisfied - see appendix D.
} 
For concreteness consider $d=4$ and $\Delta_{\chi}=2$. This corresponds to operators of dimension two in the dual field theory. Now terms in $S_{\text {loc }}$ do not have definite weight, but we can still expand

$$
\begin{aligned}
W(\phi, \chi) & =W(\phi)+X_{\alpha}(\phi) \chi^{\alpha}+\frac{1}{2} U_{\alpha \beta} \chi^{\alpha} \chi^{\beta}+\cdots, \\
-\Phi(\phi, \chi) R & =-\Phi(\phi) R+Y_{\alpha}(\phi) \chi^{\alpha} R+\cdots, \\
\frac{1}{2} M_{a b}(\phi, \chi) \partial^{i} \phi^{a} \partial_{i} \phi^{b} & =\frac{1}{2} M_{a b}(\phi) \partial^{i} \phi^{a} \partial_{i} \phi^{b}+\frac{1}{2} N_{\alpha a b}(\phi) \chi^{\alpha} \partial^{i} \phi^{a} \partial_{i} \phi^{b}+\cdots
\end{aligned}
$$

The action $S_{\text {loc }}$ now contains

$$
\mathscr{L}_{\mathrm{loc}}^{(2)}=-\Phi(\phi) R+X_{\alpha}(\phi) \chi^{\alpha}+\frac{1}{2} M_{a b}(\phi) \partial^{i} \phi^{a} \partial_{i} \phi^{b}
$$

and

$$
\mathscr{L}_{\mathrm{loc}}^{(4)}=\frac{1}{2} U_{\alpha \beta}(\phi) \chi^{\alpha} \chi^{\beta}+M_{\alpha a}(\phi) \partial^{i} \chi^{\alpha} \partial_{i} \phi^{a}+Y_{\alpha}(\phi) \chi^{\alpha} R+\frac{1}{2} N_{\alpha a b}(\phi) \chi^{\alpha} \partial^{i} \phi^{a} \partial_{i} \phi^{b} .
$$

We can also break down (4.4) by weight. As an example, writing $V(\phi, \chi)=V(\phi)+$ $V_{\alpha}(\phi) \chi^{\alpha}+\cdots$, the weight zero part of $(4.4 \mathrm{a})$ gives

$$
V=\frac{1}{2} H^{a b} \partial_{a} W \partial_{b} W-\frac{d}{4(d-1)} W^{2}+H^{\alpha a} X_{\alpha} \partial_{a} W+\frac{1}{2} H^{\alpha \beta} X_{\alpha} X_{\beta},
$$

where $V=V(\phi)$ and $W=W(\phi)$.

Note that $\mathscr{L}_{\text {loc }}^{(4)}$ includes more weight-four terms, e.g. $R^{2}$, but these correspond to ambiguities in the trace anomaly as has been explained in [9]. Contrary to this, the terms we include in (4.21) do not correspond to ambiguities, although they are of weight four. This is because the functional derivative with respect to $\chi$ that appears now in the flow equation (4.1) reduces the weight by two. This, then, modifies equations (4.4) and allows us to determine relations involving the coefficients in (4.20) and (4.21) at weight zero and two respectively. Equation (4.22) is the result at weight zero.

With (4.20) and (4.21) it is straightforward to work out the local Callan-Symanzik equation, and find holographic counterparts for the quantities considered in the case of scalar relevant operators of dimension two by Osborn [33]. For example, the left-hand side of (4.7) receives new contributions of the form

$$
\frac{2(d-1)}{W}\left(H^{\alpha \beta} \frac{1}{\sqrt{\tilde{h}}} \frac{\delta S_{\mathrm{loc}}^{(4)}}{\delta \chi^{\alpha}} \frac{1}{\sqrt{\tilde{h}}} \frac{\delta \Gamma}{\delta \chi^{\beta}}+H^{\alpha a} \frac{1}{\sqrt{\tilde{h}}} \frac{\delta S_{\mathrm{loc}}^{(2)}}{\delta \chi^{\alpha}} \frac{1}{\sqrt{\tilde{h}}} \frac{\delta \Gamma}{\delta \phi^{a}}\right)
$$

These give rise to $\Delta_{\sigma}^{m}$ and the shifts in $\hat{\Delta}_{\sigma}^{\mathrm{W}}$ and $\hat{\Delta}_{\sigma}^{\beta}$ in [33, eq. (3.25)].

If all $\chi$ 's have $\Delta_{\chi} \neq 2$, then $S_{\text {loc }}$ would include

$$
\mathscr{L}_{\mathrm{loc}}^{\left(4-\Delta_{\chi}\right)}=X_{\alpha}(\phi) \chi^{\alpha}, \quad \mathscr{L}_{\mathrm{loc}}^{\left(8-2 \Delta_{\chi}\right)}=\frac{1}{2} U_{\alpha \beta}(\phi) \chi^{\alpha} \chi^{\beta} .
$$


These will result in extra contributions to the left-hand side of (4.7) of the form

$$
\begin{gathered}
\frac{2(d-1)}{W}\left(H^{\alpha a} \frac{1}{\sqrt{\tilde{h}}} \frac{\delta S_{\mathrm{loc}}^{\left(4-\Delta_{\chi}\right)}}{\delta \phi^{a}}+H^{\alpha \beta} \frac{1}{\sqrt{\tilde{h}}} \frac{\delta S_{\mathrm{loc}}^{\left(8-2 \Delta_{\chi}\right)}}{\delta \chi^{\beta}}\right) \frac{1}{\sqrt{\tilde{h}}} \frac{\delta \Gamma}{\delta \chi^{\alpha}}= \\
\frac{2(d-1)}{W}\left(H^{\alpha a} \partial_{a} X_{\beta}+H^{\alpha \gamma} U_{\gamma \beta}\right) \chi^{\beta} \frac{1}{\sqrt{\tilde{h}}} \frac{\delta \Gamma}{\delta \chi^{\alpha}} .
\end{gathered}
$$

The quantity $\frac{2(d-1)}{W}\left(H^{\alpha a} \partial_{a} X_{\beta}+H^{\alpha \gamma} U_{\gamma \beta}\right)$ gives a holographic derivation of the operator $D^{\alpha}$ of [37, eq. (2.12)].

Finally, we note here that dimension three vector operators in the boundary theory can also be added by considering gauge fields in the bulk. A discussion of this can be found in [49].

\section{Acknowledgments}

We would like to thank Hong Liu for many valuable discussions and suggestions. AS would also like to thank Hugh Osborn and Guilherme Pimentel for insightful comments when he presented part of this work at DAMTP, University of Cambridge, and Kostas Skenderis for helpful discussions. For our computations we have relied on Mathematica and the package xAct. AS is grateful to the Aspen Center for Physics (partially supported by National Science Foundation Grant No. 1066293), for hospitality during the final stages of this project. SR is supported by the U.S. Department of Energy under cooperative research agreement Contract No. DE-FG02-05ER41360. The research of AS is supported in part by the National Science Foundation under Grant No. 1350180. YZ is supported by ARO under Grant No. W911NF-12-0486.

\section{A ADM formalism}

We consider a $(d+1)$-dimensional manifold with coordinates $x^{\mu}$ and metric $\tilde{g}_{\mu \nu}$. We will work in Euclidean signature so that locally $\tilde{g}_{\mu \nu}=\delta_{\mu \nu}$, where $\delta_{\mu \nu}$ is the Kronecker delta. The line element can be written in the ADM form [45]

$$
d s^{2}=\tilde{g}_{\mu \nu} d x^{\mu} d x^{\nu}=N^{2}(y, r) d r^{2}+\tilde{h}_{i j}\left(d y^{i}+N^{i}(y, r) d r\right)\left(d y^{j}+N^{j}(y, r) d r\right),
$$

where we assume a hypersurface-foliation of the $(d+1)$-dimensional spacetime along the radial coordinate $r$, and we define

$$
\tilde{h}_{i j}=\tilde{g}_{\mu \nu} e_{i}^{\mu} e_{j}^{\nu}, \quad e_{i}^{\mu}=\frac{\partial x^{\mu}}{\partial y^{i}},
$$

as the induced metric and $y$ as the coordinates on the hypersurfaces, while $N$ is the lapse function and $N^{i}$ the shift vector. We also define the vector

$$
t^{\mu}=\frac{\partial x^{\mu}}{\partial r}=N n^{\mu}+N^{i} e_{i}^{\mu}
$$


where $n^{\mu}$ is a vector normal to the hypersurfaces with $n^{\mu} n_{\mu}=1$. The inverse of $\tilde{g}_{\mu \nu}$ is given by

$$
\tilde{g}^{\mu \nu}=\tilde{h}^{i j} e_{i}^{\mu} e_{j}^{\nu}+n^{\mu} n^{\nu}
$$

where $\tilde{h}^{i j}$ is the inverse of $\tilde{h}_{i j}$.

The starting point for the decomposition of curvature tensors is the Gauss-Weingarten equation,

$$
\nabla_{\nu} e_{i}^{\mu} e_{j}^{\nu}=\Gamma_{i j}^{k} e_{k}^{\mu}-K_{i j} n^{\mu}
$$

as well as the equations

$$
\begin{aligned}
& \nabla^{\mu} n_{\nu} e_{i}^{\nu}=K_{i}^{j} e_{j}^{\mu}+a_{i} n^{\mu}, \\
& \nabla_{\nu} n^{\mu} e_{i}^{\nu}=K_{i}^{j} e_{j}^{\mu},
\end{aligned}
$$

where

$$
K_{i j}=\nabla_{(\mu} n_{\nu)} e_{i}^{\mu} e_{j}^{\nu}=\frac{1}{2} £_{n} \tilde{g}_{\mu \nu} e_{i}^{\mu} e_{j}^{\nu},
$$

with $£_{n}$ the Lie derivative along $n^{\mu}$, is the extrinsic curvature, $\Gamma_{j k}^{i}$ is the Christoffel symbol defined from $\tilde{h}_{i j}$, and $a_{i}=a_{\mu} e_{i}^{\mu}$ with $a_{\mu}=\nabla_{\nu} n_{\mu} n^{\nu}$. With the help of (A.5) and (A.6), and with the definition $\left[\nabla_{\mu}, \nabla_{\nu}\right] A^{\rho}=R_{\sigma \mu \nu}^{\rho} A^{\sigma}$ for the Riemann tensor, we can derive

$$
\begin{aligned}
& R_{\mu \nu \rho \sigma} e_{i}^{\mu} e_{j}^{\nu} e_{k}^{\rho} e_{l}^{\sigma}=R_{i j k l}-K_{i k} K_{j l}+K_{i l} K_{j k}, \\
& R_{\mu \nu \rho \sigma} n^{\mu} e_{i}^{\nu} e_{j}^{\rho} e_{k}^{\sigma}=\nabla_{k} K_{i j}-\nabla_{j} K_{i k}, \\
& R_{\mu \nu \rho \sigma} n^{\mu} e_{i}^{\nu} n^{\rho} e_{j}^{\sigma}=-£_{n} K_{i j}+K_{i k} K_{j}^{k}+\nabla_{i} a_{j}-a_{i} a_{j},
\end{aligned}
$$

where $£_{n} K_{i j} \equiv £_{n} K_{\mu \nu} e_{i}^{\mu} e_{j}^{\nu}, K_{\mu \nu}=\nabla_{\mu} n_{\nu}-n_{\mu} a_{\nu}$, and $\nabla_{i} a_{j} \equiv \nabla_{\mu} a_{\nu} e_{i}^{\mu} e_{j}^{\nu}$. Equations (A.8a) and (A.8b) are known as the Gauss-Codazzi equations, while equation (A.8c) is known as the Ricci equation. The decomposition of the Ricci tensor $R_{\mu \nu}=R_{\mu \rho \nu}^{\rho}$ is given by

$$
\begin{aligned}
R_{\mu \nu} e_{i}^{\mu} e_{j}^{\nu} & =R_{i j}-£_{n} K_{i j}-K K_{i j}+2 K_{i}^{k} K_{k j}+\nabla_{i} a_{j}-a_{i} a_{j}, \\
R_{\mu \nu} n^{\mu} e_{i}^{\nu} & =-\partial_{i} K+\nabla_{j} K_{i}^{j} \\
R_{\mu \nu} n^{\mu} n^{\nu} & =-\tilde{h}^{i j} £_{n} K_{i j}+K^{i j} K_{i j}+\nabla^{i} a_{i}-a^{i} a_{i} .
\end{aligned}
$$

Finally, the Ricci scalar $R=\tilde{g}^{\mu \nu} R_{\mu \nu}$ has the decomposition

$$
R={ }^{d} R-2 \tilde{h}^{i j} £_{n} K_{i j}-K^{2}+3 K^{i j} K_{i j}+2\left(\nabla^{i} a_{i}-a^{i} a_{i}\right) .
$$

In terms of the lapse it is not hard to see that $a_{i}=-\partial_{i} \log N$, and so

$$
\nabla_{i} a_{j}-a_{i} a_{j}=-\frac{1}{N} \nabla_{i} \partial_{j} N
$$

It is now possible to express some of the above quantities using the Lie derivative of the vector $t^{\mu}$ of (A.3) as opposed to $n^{\mu}$, as well as the lapse and shift, since $£_{t}=N £_{n}+£_{N}$. From (A.3) we see that $£_{t}=\partial / \partial r$ on a scalar. We define the tensor

$$
L_{i j}=£_{n} K_{i j}+\frac{1}{N} \nabla_{i} \partial_{j} N=\frac{1}{N}\left(\dot{K}_{i j}-£_{N} K_{i j}+\nabla_{i} \partial_{j} N\right), \quad \dot{K}_{i j} \equiv £_{t} K_{i j},
$$


with the aid of which we can express (A.8c) as

$$
R_{\mu \nu \rho \sigma} n^{\mu} e_{i}^{\nu} n^{\rho} e_{j}^{\sigma}=K_{i}^{k} K_{k j}-L_{i j},
$$

and also (A.9a), (A.9c) and (A.10) as

$$
\begin{aligned}
R_{\mu \nu} e_{i}^{\mu} e_{j}^{\nu} & =R_{i j}-K K_{i j}+2 K_{i}^{k} K_{k j}-L_{i j}, \\
R_{\mu \nu} n^{\mu} n^{\nu} & =K^{i j} K_{i j}-L, \quad L=\tilde{h}^{i j} L_{i j},
\end{aligned}
$$

and

$$
R={ }^{d} R-K^{2}+3 K^{i j} K_{i j}-2 L .
$$

Now, from (A.7) we find

$$
\dot{\tilde{h}}_{i j} \equiv £_{t} \tilde{h}_{i j}=2 N K_{i j}+\nabla_{i} N_{j}+\nabla_{j} N_{i},
$$

and we can also compute

$$
£_{t} \sqrt{\tilde{h}}=\sqrt{\tilde{h}}\left(N K+\nabla^{i} N_{i}\right) .
$$

Finally,

$$
L=\frac{1}{N}\left(\frac{1}{\sqrt{\tilde{h}}} £_{t}(\sqrt{\tilde{h}} K)+\nabla^{i}\left(\partial_{i} N-K N_{i}\right)\right)-K^{2}+2 K^{i j} K_{i j},
$$

and so we can write

$$
R={ }^{d} R+K^{2}-K^{i j} K_{i j}-\frac{2}{N}\left(\frac{1}{\sqrt{\tilde{h}}} £_{t}(\sqrt{\tilde{h}} K)+\nabla^{i}\left(\partial_{i} N-K N_{i}\right)\right) .
$$

With (A.20) we can easily read off the Gibbons-Hawking-York term for Einstein gravity $[42,43]$.

\section{B Boundary terms}

In this appendix we work out the allowed $\phi$-dependent boundary terms $\mathscr{L}_{\partial}^{\phi}$ in (2.4). The form of $\mathscr{L}_{\partial}^{\tilde{h}}$ has been determined in [15].

The transformation properties of a general symmetric two-index tensor $S_{\mu \nu}$ with the ADM decomposition

$$
S_{\mu \nu} d x^{\mu} d x^{\nu}=F(y, r) d r^{2}+2 G_{i}(y, r) d r d y^{i}+H_{i j}(y, r) d y^{i} d y^{j}
$$

under the infinitesimal transformation considered in [44], namely

$$
\begin{gathered}
r \rightarrow r^{\prime}=r+\epsilon(y, r), \\
y^{i} \rightarrow y^{i}=y^{i}+\epsilon^{i}(y, r),
\end{gathered}
$$

can be seen as follows. ${ }^{7}$ Under a change of coordinates and demanding that $S_{\mu \nu}$ be invariant we get the condition

$$
F d r^{2}+2 G_{i} d r d y^{i}+H_{i j} d y^{i} d y^{j}=F^{\prime} d r^{2}+2 G_{i}^{\prime} d r^{\prime} d y^{i}+H_{i j}^{\prime} d y^{\prime i} d y^{\prime j},
$$

\footnotetext{
${ }^{7}$ Note that under a finite diffeomorphism of the form $x^{\mu} \rightarrow f^{\mu}(x)$ we require $f^{r}\left(y, r_{0}\right)=r_{0}$ so that the location of the boundary is fixed.
} 
where the primed quantities on the right-hand side are functions of $r^{\prime}, y^{\prime}$. Also,

$$
\begin{aligned}
d r^{\prime} & =d r+\partial_{r} \epsilon d r+\partial_{i} \epsilon d y^{i}, \\
d y^{\prime i} & =d y^{i}+\partial_{r} \epsilon^{i} d r+\partial_{j} \epsilon^{i} d y^{j},
\end{aligned}
$$

and from the first equation we see that when $d r=0$ then $d r^{\prime}=0$ only if $\partial_{i} \epsilon=0$. This is a condition we require only on the boundary, and thus $\partial_{i} \epsilon\left(y, r_{0}\right)=0$. Keeping only terms involving the derivatives of $\epsilon, \epsilon^{i}$, we get

$$
\begin{aligned}
F^{\prime} & =F-2 F \partial_{r} \epsilon-2 G_{i} \partial_{r} \epsilon^{i}, \\
G_{i}^{\prime} & =G_{i}-F \partial_{i} \epsilon-G_{i} \partial_{r} \epsilon-G_{j} \partial_{i} \epsilon^{j}-H_{i j} \partial_{r} \epsilon^{j}, \\
H_{i j}^{\prime} & =H_{i j}-G_{i} \partial_{j} \epsilon-G_{j} \partial_{i} \epsilon-H_{i k} \partial_{j} \epsilon^{k}-H_{j k} \partial_{i} \epsilon^{k} .
\end{aligned}
$$

As a check, we note that taking $H_{i j}=\tilde{h}_{i j}, F=N^{2}+\tilde{h}_{i j} N^{i} N^{j}$ and $G_{i}=N_{i}$ reproduces equation (C.2) of [44] for the transformation of the lapse, shift and induced metric.

We now apply this procedure to the tensor $\partial_{\mu} \phi \partial_{\nu} \phi$, which has the ADM form

$$
\partial_{\mu} \phi \partial_{\nu} \phi=\left(N £_{n} \phi+£_{N} \phi\right)^{2} N^{2} d r^{2}+2\left(N £_{n} \phi+£_{N} \phi\right) \partial_{i} \phi d r d y^{i}+\partial_{i} \phi \partial_{j} \phi d y^{i} d y^{j},
$$

and get

$$
\delta\left(\partial_{i} \phi \partial_{j} \phi\right)=-\partial_{i} \phi \partial_{k} \phi \partial_{j} \epsilon^{k}-\partial_{j} \phi \partial_{k} \phi \partial_{i} \epsilon^{k} .
$$

Also, if we use $\partial_{i} \epsilon\left(y, r_{0}\right)=0$ then

$$
\delta K_{i j}=-\partial_{i} \epsilon^{k} K_{k j}-\partial_{j} \epsilon^{k} K_{k i}
$$

and so

$$
\delta K^{i j}=-\left(\tilde{h}^{i k} K^{j l}+g^{j k} K^{i l}\right) \delta \tilde{h}_{k l}+g^{i k} g^{j l} \delta K_{j l}=K^{i k} \partial_{k} \epsilon^{j}+K^{j k} \partial_{k} \epsilon^{i} .
$$

Thus, with the use of (B.7) and (B.9) we finally find

$$
\delta\left(K^{i j} \partial_{i} \phi \partial_{j} \phi\right)=0,
$$

which shows that $K^{i j} \partial_{i} \phi \partial_{j} \phi$ is an allowed boundary term. Furthermore, since $\delta K=0$ and $\delta\left(\partial^{i} \phi \partial_{i} \phi\right)=0$, we conclude that $K \partial^{i} \phi \partial_{i} \phi$ is also allowed.

There is a further term that is quadratic in $\phi$, has three derivatives at the boundary and one of them is a radial derivative, namely $\partial^{i} £_{n} \phi \partial_{i} \phi$, or, equivalently, $£_{n} \phi \nabla^{2} \phi$. The transformation property of the Lie derivative can be worked out by considering

$$
d \phi=\left(N £_{n} \phi+£_{N} \phi\right) d r+\partial_{i} \phi d y^{i}=\left(N £_{n} \phi+£_{N} \phi\right)^{\prime} d r^{\prime}+\left(\partial_{i} \phi\right)^{\prime} d y^{i},
$$

which gives

$$
\delta\left(N £_{n} \phi\right)=-N £_{n} \phi \partial_{r} \epsilon \Rightarrow \delta\left(£_{n} \phi\right)=0 .
$$

Furthermore, we have $\delta\left(\nabla_{i} \partial_{j} \phi\right)=-\nabla_{i} \partial_{k} \phi \partial_{j} \epsilon^{k}-\nabla_{j} \partial_{k} \phi \partial_{i} \epsilon^{k}$ and so

$$
\delta\left(\nabla^{2} \phi\right)=0 .
$$

Thus, from (B.12) and (B.13) we see that the term $£_{n} \phi \nabla^{2} \phi$ is an allowed boundary term. Note that the difference between the terms $\nabla^{2} \phi \nabla^{2} \phi$ and $\nabla^{\mu} \partial^{\nu} \phi \nabla_{\mu} \partial_{\nu} \phi$ in (2.2) contains just the boundary terms in $\mathscr{L}_{\partial}^{\phi}$ of (2.4). Nevertheless, the coefficient of $\nabla^{\mu} \partial^{\nu} \phi \nabla_{\mu} \partial_{\nu} \phi$ in (2.2) contributes to the anomaly (3.14) while that of $\nabla^{2} \phi \nabla^{2} \phi$ does not. 


\section{Expressions for six-dimensional curvature tensors}

A complete basis of scalar dimension-six curvature terms consists of [50]

$$
\begin{aligned}
& K_{1}=R^{3}, \quad K_{2}=R R^{i j} R_{i j}, \quad K_{3}=R R^{i j k l} R_{i j k l}, \quad K_{4}=R^{i j} R_{j k} R_{i}^{k}, \\
& K_{5}=R^{i j} R^{k l} R_{i k l j}, \quad K_{6}=R^{i j} R_{i k l m} R_{j}^{k l m}, \quad K_{7}=R^{i j k l} R_{k l m n} R^{m n}{ }_{i j}, \\
& K_{8}=R^{i j k l} R_{m j k n} R_{i}^{m n}{ }_{l}, \quad K_{9}=R \nabla^{2} R, \quad K_{10}=R^{i j} \nabla^{2} R_{i j}, \quad K_{11}=R^{i j k l} \nabla^{2} R_{i j k l}, \\
& K_{12}=R^{i j} \nabla_{i} \partial_{j} R, \quad K_{13}=\nabla^{i} R^{j k} \nabla_{i} R_{j k}, \quad K_{14}=\nabla^{i} R^{j k} \nabla_{j} R_{i k}, \\
& K_{15}=\nabla^{i} R^{j k l m} \nabla_{i} R_{j k l m}, \quad K_{16}=\nabla^{2} R^{2}, \quad K_{17}=\left(\nabla^{2}\right)^{2} R .
\end{aligned}
$$

In equation (3.14) in the main text we use the combinations

$$
\begin{aligned}
I_{1}= & \frac{19}{800} K_{1}-\frac{57}{160} K_{2}+\frac{3}{40} K_{3}+\frac{7}{16} K_{4}-\frac{9}{8} K_{5}-\frac{3}{4} K_{6}+K_{8}, \\
I_{2}= & \frac{9}{200} K_{1}-\frac{27}{40} K_{2}+\frac{3}{10} K_{3}+\frac{5}{4} K_{4}-\frac{3}{2} K_{5}-3 K_{6}+K_{7}, \\
I_{3}= & -\frac{11}{50} K_{1}+\frac{27}{10} K_{2}-\frac{6}{5} K_{3}-K_{4}+6 K_{5}+2 K_{7}-8 K_{8} \\
& +\frac{3}{5} K_{9}-6 K_{10}+6 K_{11}+3 K_{13}-6 K_{14}+3 K_{15}, \\
E_{6}= & K_{1}-12 K_{2}+3 K_{3}+16 K_{4}-24 K_{5}-24 K_{6}+4 K_{7}+8 K_{8}, \\
J_{1}= & 6 K_{6}-3 K_{7}+12 K_{8}+K_{10}-7 K_{11}-11 K_{13}+12 K_{14}-4 K_{15}, \\
J_{2}= & -\frac{1}{5} K_{9}+K_{10}+\frac{2}{5} K_{12}+K_{13}, \\
J_{3}= & K_{4}+K_{5}-\frac{3}{20} K_{9}+\frac{4}{5} K_{12}+K_{14}, \\
J_{4}= & -\frac{1}{5} K_{9}+K_{11}+\frac{2}{5} K_{12}+K_{15} .
\end{aligned}
$$

\section{Anomaly coefficients and consistency conditions in $d=4$}

Osborn's consistency conditions in $d=4$ can all be verified using the holographic result (4.9). First, we list here the holographic results for the coefficients in (4.12). We have

$$
\begin{gathered}
A=C=-\frac{1}{2 \tilde{\kappa}_{5}^{2}} \frac{3}{W} \Phi^{2}, \quad B=\frac{1}{2 \tilde{\kappa}_{5}^{2}} \frac{27}{W} \partial_{a} \Phi \partial_{b} \Phi H^{a b}, \quad D=0, \\
E_{a}^{\phi}=-\frac{1}{2 \tilde{\kappa}_{5}^{2}} \frac{18}{W} M_{a b} \partial_{c} \Phi H^{c d}, \quad W_{a}^{\phi}=\frac{1}{2 \tilde{\kappa}_{5}^{2}} \frac{12}{W} \Phi \partial_{a} \Phi, \quad Y_{a}^{\phi}=-\frac{1}{2 \tilde{\kappa}_{5}^{2}} \frac{18}{W} M_{a b} \partial_{c} \Phi H^{b c}, \quad U_{a}^{\phi}=0,
\end{gathered}
$$

$$
\begin{aligned}
F_{a b}^{\phi}= & -\frac{1}{2 \tilde{\kappa}_{5}^{2}} \frac{6}{W}\left(M_{a b} \Phi+3 \partial_{a} \Phi \partial_{b} \Phi+3 \partial_{c} M_{a b} \partial_{d} \Phi H^{c d}-6 M_{c(a} \partial_{b)} \log W \partial_{d} \Phi H^{c d}\right. \\
& \left.+6 M_{c(a} \partial_{b)} \partial_{d} \Phi H^{c d}+6 M_{c(a} \partial_{b)} H^{c d} \partial_{d} \Phi\right), \\
G_{a b}^{\phi}= & -\frac{1}{2 \tilde{\kappa}_{5}^{2}} \frac{12}{W}\left(\left(M_{a b}+\partial_{a} \log W \partial_{b} \Phi+\partial_{b} \log W \partial_{a} \Phi\right) \Phi-\partial_{a} \Phi \partial_{b} \Phi\right), \\
A_{a b}^{\phi}= & \frac{1}{2 \tilde{\kappa}_{5}^{2}} \frac{6}{W} M_{a c} M_{b d} H^{c d}, \quad V_{a b}^{\phi}=-\frac{1}{2 \tilde{\kappa}_{5}^{2}} \frac{6}{W} \partial_{a} \Phi \partial_{b} \Phi, \quad S_{a b}^{\phi}=\frac{1}{2 \tilde{\kappa}_{5}^{2}} \frac{6}{W} \partial_{a} \Phi \partial_{b} \Phi,
\end{aligned}
$$




$$
\begin{aligned}
B_{a b c}^{\phi} & =-\frac{1}{2 \tilde{\kappa}_{5}^{2}} \frac{6}{W}\left(\partial_{a} \Phi \partial_{b} \Phi \partial_{c} \log W+2 \partial_{c} \Phi \partial_{(a} \Phi \partial_{b)} \log W+2 M_{c(a} \partial_{b)} \Phi-2 \Gamma_{a b d}^{M} M_{c e} H^{d e}\right), \\
T_{a b c}^{\phi} & =\frac{1}{2 \tilde{\kappa}_{5}^{2}} \frac{6}{W}\left(M_{a b} \partial_{c} \Phi-2 \partial_{a} \Phi \partial_{b} \Phi \partial_{c} \log W+2 \partial_{a} \partial_{b} \Phi \partial_{c} \Phi-2 M_{c(a} \partial_{b)} \Phi\right),
\end{aligned}
$$

and

$$
\begin{aligned}
C_{a b c d}^{\phi}= & -\frac{1}{2 \tilde{\kappa}_{5}^{2}} \frac{2}{W}\left(M_{a b} M_{c d}-3 M_{a(c} M_{d) b}\right. \\
& +3 M_{a b} \partial_{(c} \Phi \partial_{d)} \log W+3 M_{c d} \partial_{(a} \Phi \partial_{b)} \log W \\
& -3 M_{a c} \partial_{(b} \Phi \partial_{d)} \log W-3 M_{b c} \partial_{(a} \Phi \partial_{d)} \log W \\
& -3 M_{a d} \partial_{(b} \Phi \partial_{c)} \log W-3 M_{b d} \partial_{(a} \Phi \partial_{c)} \log W \\
& -3 \partial_{a} \Phi \partial_{b} \Phi \partial_{c} \log W \partial_{d} \log W-3 \partial_{c} \Phi \partial_{d} \Phi \partial_{a} \log W \partial_{b} \log W \\
& +6 \partial_{a} \partial_{b} \Phi \partial_{(c} \Phi \partial_{d)} \log W+6 \partial_{c} \partial_{d} \Phi \partial_{(a} \Phi \partial_{b)} \log W \\
& +3 \partial_{a} \Phi \partial_{b} \Phi \partial_{c} \partial_{d} \log W+3 \partial_{c} \Phi \partial_{d} \Phi \partial_{a} \partial_{b} \log W \\
& \left.+6 \partial_{d} M_{c(a} \partial_{b)} \Phi+6 \Gamma_{a b(c}^{M} \partial_{d)} \Phi-6 \partial_{(a} \Phi \Gamma_{b) c d}^{M}-6 \Gamma_{a b e}^{M} \Gamma_{c d f}^{M} H^{e f}\right)
\end{aligned}
$$

With these results and with the use of $(4.4 \mathrm{~b}),(4.4 \mathrm{c}),(4.4 \mathrm{~d})$, and $(4.5)$ we find that the consistency conditions of [33], namely

$$
\begin{aligned}
8 \partial_{a} A-G_{a b}^{\phi} \beta^{b} & =-£_{\beta} W_{a}^{\phi}, \\
2 E_{i}^{\phi}+A_{a b}^{\phi} \beta^{b} & =-£_{\beta} U_{a}^{\phi}, \\
8 B-A_{a b}^{\phi} \beta^{a} \beta^{b} & =£_{\beta}\left(2 D+U_{a}^{\phi} \beta^{a}\right), \\
4 \partial_{a} B+\left(A_{a b}^{\phi}+F_{a b}^{\phi}\right) \beta^{b} & =£_{\beta}\left(\partial_{a} D+Y_{a}^{\phi}-U_{a}^{\phi}\right), \\
G_{a b}^{\phi}+2 A_{a b}^{\phi}+\Lambda_{a b}^{\phi} & =£_{\beta} S_{a b}^{\phi}, \quad \Lambda_{a b}^{\phi}=2 \partial_{a} \beta^{c} A_{c b}^{\phi}+\beta^{c} B_{c a b}^{\phi}, \\
2\left(A_{a b}^{\phi}+F_{a b}^{\phi}\right)+\Lambda_{a b}^{\phi}+\beta^{c}\left(2 \bar{A}_{c(a b)}^{\phi}-\bar{A}_{a b c}^{\phi}\right) & =£_{\beta}\left(S_{a b}^{\phi}-A_{a b}^{\phi}-2 \partial_{(a} U_{b)}^{\phi}+V_{a b}^{\phi}\right), \\
\bar{A}_{a b c}^{\phi} & =\partial_{c} A_{a b}^{\phi}-B_{c(a b)}^{\phi}, \\
\partial_{(a} G_{b) c}^{\phi}-\frac{1}{2} \partial_{c} G_{a b}^{\phi}+B_{a b c}^{\phi}+\partial_{c} \beta^{d} B_{a b d}^{\phi}+C_{a b c d}^{\phi} \beta^{d} & =\frac{1}{2} £_{\beta} T_{a b c}^{\phi}+\partial_{a} \partial_{b} \beta^{d} S_{c d}^{\phi},
\end{aligned}
$$

are satisfied.

Open Access. This article is distributed under the terms of the Creative Commons Attribution License (CC-BY 4.0), which permits any use, distribution and reproduction in any medium, provided the original author(s) and source are credited.

\section{References}

[1] D.M. Capper and M.J. Duff, Trace anomalies in dimensional regularization, Nuovo Cim. A 23 (1974) 173 [INSPIRE].

[2] M.J. Duff, Twenty years of the Weyl anomaly, Class. Quant. Grav. 11 (1994) 1387 [hep-th/9308075] [INSPIRE]. 
[3] J.M. Maldacena, The Large-N limit of superconformal field theories and supergravity, Int. J. Theor. Phys. 38 (1999) 1113 [hep-th/9711200] [INSPIRE].

[4] S.S. Gubser, I.R. Klebanov and A.M. Polyakov, Gauge theory correlators from noncritical string theory, Phys. Lett. B 428 (1998) 105 [hep-th/9802109] [INSPIRE].

[5] E. Witten, Anti-de Sitter space and holography, Adv. Theor. Math. Phys. 2 (1998) 253 [hep-th/9802150] [INSPIRE].

[6] M. Henningson and K. Skenderis, The Holographic Weyl anomaly, JHEP 07 (1998) 023 [hep-th/9806087] [INSPIRE].

[7] J. de Boer, E.P. Verlinde and H.L. Verlinde, On the holographic renormalization group, JHEP 08 (2000) 003 [hep-th/9912012] [INSPIRE].

[8] J. de Boer, The Holographic renormalization group, Fortsch. Phys. 49 (2001) 339 [hep-th/0101026] [INSPIRE].

[9] M. Fukuma, S. Matsuura and T. Sakai, A Note on the Weyl anomaly in the holographic renormalization group, Prog. Theor. Phys. 104 (2000) 1089 [hep-th/0007062] [INSPIRE].

[10] S. Nojiri, S.D. Odintsov and S. Ogushi, Holographic renormalization group and conformal anomaly for AdS $S_{9} / C F T_{8}$ correspondence, Phys. Lett. B 500 (2001) 199 [hep-th/0011182] [INSPIRE].

[11] I. Papadimitriou and K. Skenderis, AdS/CFT correspondence and geometry, hep-th/0404176 [INSPIRE].

[12] I. Papadimitriou and K. Skenderis, Correlation functions in holographic RG flows, JHEP 10 (2004) 075 [hep-th/0407071] [INSPIRE].

[13] S. Nojiri and S.D. Odintsov, On the conformal anomaly from higher derivative gravity in AdS/CFT correspondence, Int. J. Mod. Phys. A 15 (2000) 413 [hep-th/9903033] [InSPIRE].

[14] M. Blau, K.S. Narain and E. Gava, On subleading contributions to the AdS/CFT trace anomaly, JHEP 09 (1999) 018 [hep-th/9904179] [INSPIRE].

[15] M. Fukuma, S. Matsuura and T. Sakai, Higher derivative gravity and the AdS/CFT correspondence, Prog. Theor. Phys. 105 (2001) 1017 [hep-th/0103187] [INSPIRE].

[16] I. Papadimitriou, Holographic Renormalization of general dilaton-axion gravity, JHEP 08 (2011) 119 [arXiv:1106.4826] [INSPIRE].

[17] V. Jahnke, A.S. Misobuchi and D. Trancanelli, Holographic renormalization and anisotropic black branes in higher curvature gravity, JHEP 01 (2015) 122 [arXiv:1411.5964] [INSPIRE].

[18] S. de Haro, S.N. Solodukhin and K. Skenderis, Holographic reconstruction of space-time and renormalization in the AdS/CFT correspondence, Commun. Math. Phys. 217 (2001) 595 [hep-th/0002230] [INSPIRE].

[19] S.M. Paneitz, A quartic conformally covariant differential operator for arbitrary pseudo-Riemannian manifolds (summary), SIGMA 4 (2008) 36 [arXiv:0803.4331].

[20] E.S. Fradkin and A.A. Tseytlin, One Loop $\beta$-function in Conformal Supergravities, Nucl. Phys. B 203 (1982) 157 [INSPIRE].

[21] E.S. Fradkin and A.A. Tseytlin, Asymptotic freedom in extended conformal supergravities, Phys. Lett. B 110 (1982) 117 [INSPIRE]. 
[22] E.S. Fradkin and A.A. Tseytlin, Conformal Anomaly in Weyl Theory and Anomaly Free Superconformal Theories, Phys. Lett. B 134 (1984) 187 [INSPIRE].

[23] R.J. Riegert, A Nonlocal Action for the Trace Anomaly, Phys. Lett. B 134 (1984) 56 [INSPIRE].

[24] I. Jack and H. Osborn, Constraints on RG Flow for Four Dimensional Quantum Field Theories, Nucl. Phys. B 883 (2014) 425 [arXiv:1312.0428] [InSPIRE].

[25] M. Bianchi, D.Z. Freedman and K. Skenderis, Holographic renormalization, Nucl. Phys. B 631 (2002) 159 [hep-th/0112119] [INSPIRE].

[26] T.P. Branson, Differential operators cononically associated to a conformal structure, Math. Scand. 57 (1985) 293.

[27] H. Osborn and A. Stergiou, Structures on the Conformal Manifold in Six Dimensional Theories, JHEP 04 (2015) 157 [arXiv:1501.01308] [INSPIRE].

[28] D.M. Hofman and J.M. Maldacena, Conformal collider physics: energy and charge correlations, JHEP 05 (2008) 012 [arXiv:0803.1467] [INSPIRE].

[29] D.M. Hofman, Higher Derivative Gravity, Causality and Positivity of Energy in a UV complete QFT, Nucl. Phys. B 823 (2009) 174 [arXiv:0907.1625] [InSPIRE].

[30] J. de Boer, M. Kulaxizi and A. Parnachev, $A d S_{7} / C F T_{6}$, Gauss-Bonnet Gravity and Viscosity Bound, JHEP 03 (2010) 087 [arXiv:0910.5347] [INSPIRE].

[31] X.O. Camanho and J.D. Edelstein, Causality constraints in AdS/CFT from conformal collider physics and Gauss-Bonnet gravity, JHEP 04 (2010) 007 [arXiv:0911.3160] [INSPIRE].

[32] A. Buchel, J. Escobedo, R.C. Myers, M.F. Paulos, A. Sinha and M. Smolkin, Holographic GB gravity in arbitrary dimensions, JHEP 03 (2010) 111 [arXiv:0911.4257] [INSPIRE].

[33] H. Osborn, Weyl consistency conditions and a local renormalization group equation for general renormalizable field theories, Nucl. Phys. B 363 (1991) 486 [INSPIRE].

[34] I. Jack and H. Osborn, Analogs for the $c$ Theorem for Four-dimensional Renormalizable Field Theories, Nucl. Phys. B 343 (1990) 647 [InSPIRE].

[35] B. Grinstein, A. Stergiou, D. Stone and M. Zhong, Two-loop renormalization of multiflavor $\phi^{3}$ theory in six dimensions and the trace anomaly, Phys. Rev. D 92 (2015) 045013 [arXiv: 1504.05959] [INSPIRE].

[36] J. Wess and B. Zumino, Consequences of anomalous Ward identities, Phys. Lett. B 37 (1971) 95 [INSPIRE].

[37] F. Baume, B. Keren-Zur, R. Rattazzi and L. Vitale, The local Callan-Symanzik equation: structure and applications, JHEP 08 (2014) 152 [arXiv: 1401.5983] [INSPIRE].

[38] B. Grinstein, A. Stergiou and D. Stone, Consequences of Weyl Consistency Conditions, JHEP 11 (2013) 195 [arXiv: 1308.1096] [INSPIRE].

[39] A.B. Zamolodchikov, Irreversibility of the Flux of the Renormalization Group in a $2 D$ Field Theory, JETP Lett. 43 (1986) 730 [INSPIRE].

[40] B. Grinstein, D. Stone, A. Stergiou and M. Zhong, Challenge to the a Theorem in Six Dimensions, Phys. Rev. Lett. 113 (2014) 231602 [arXiv:1406.3626] [INSPIRE]. 
[41] J. Erdmenger, A Field theoretical interpretation of the holographic renormalization group, Phys. Rev. D 64 (2001) 085012 [hep-th/0103219] [INSPIRE].

[42] G.W. Gibbons and S.W. Hawking, Action Integrals and Partition Functions in Quantum Gravity, Phys. Rev. D 15 (1977) 2752 [inSPIRE].

[43] J.W. York Jr., Role of conformal three geometry in the dynamics of gravitation, Phys. Rev. Lett. 28 (1972) 1082 [INSPIRE].

[44] M. Fukuma, S. Matsuura and T. Sakai, Holographic renormalization group, Prog. Theor. Phys. 109 (2003) 489 [hep-th/0212314] [INSPIRE].

[45] R.L. Arnowitt, S. Deser and C.W. Misner, The Dynamics of general relativity, Gen. Rel. Grav. 40 (2008) 1997 [gr-qc/0405109] [INSPIRE].

[46] F. Bastianelli, S. Frolov and A.A. Tseytlin, Conformal anomaly of $(2,0)$ tensor multiplet in six-dimensions and AdS/CFT correspondence, JHEP 02 (2000) 013 [hep-th/0001041] [INSPIRE].

[47] D. Anselmi, L. Girardello, M. Porrati and A. Zaffaroni, A Note on the holographic beta and C functions, Phys. Lett. B 481 (2000) 346 [hep-th/0002066] [INSPIRE].

[48] X.O. Camanho, J.D. Edelstein, J.M. Maldacena and A. Zhiboedov, Causality Constraints on Corrections to the Graviton Three-Point Coupling, arXiv:1407.5597 [INSPIRE].

[49] Y. Nakayama, Vector $\beta$-function, Int. J. Mod. Phys. A 28 (2013) 1350166 [arXiv: 1310.0574] [INSPIRE].

[50] L. Bonora, P. Pasti and M. Bregola, Weyl cocycles, Class. Quant. Grav. 3 (1986) 635 [INSPIRE]. 